\title{
DISTRIBUTED OPTIMAL CONTROL OF THE CAHN-HILLIARD SYSTEM INCLUDING THE CASE OF A DOUBLE-OBSTACLE HOMOGENEOUS FREE ENERGY DENSITY*
}

\author{
M. HINTERMÜLLER ${ }^{\dagger}$ AND D. WEGNER ${ }^{\ddagger}$
}

\begin{abstract}
In this paper we study the distributed optimal control for the Cahn-Hilliard system. A general class of free energy potentials is allowed which, in particular, includes the double-obstacle potential. The latter potential yields an optimal control problem of a parabolic variational inequality which is of fourth order in space. We show the existence of optimal controls to approximating problems where the potential is replaced by a mollified version of its Moreau-Yosida approximation. Corresponding first-order optimality conditions for the mollified problems are given. For this purpose a new result on the continuous Fréchet differentiability of superposition operators with values in Sobolev spaces is established. Besides the convergence of optimal controls of the mollified problems to an optimal control of the original problem, we also derive first-order optimality conditions for the original problem by a limit process. The newly derived stationarity system corresponds to a function space version of C-stationarity.
\end{abstract}

Key words. Cahn-Hilliard system, double-obstacle potential, mathematical programming with equilibrium constraints, distributed optimal control, Yosida regularization, C-stationarity

AMS subject classifications. 35K86, 49J20, 34G25

DOI. $10.1137 / 110824152$

1. Introduction. In the study of interface dynamics phase field models received a considerable amount of attention in the recent past; see, e.g., [58] for a review on phase field models in material science. Such models have also been applied successfully in fluid dynamics [7], image processing [15,22], and cancer growth modeling [28].

In [21] Cahn and Hilliard introduced a continuous model for phase transitions in systems of nonuniform compositions capturing spinodal decomposition. Based on the minimization of a Ginzburg-Landau type free energy, the resulting system is of parabolic type with a fourth-order (partial) differential operator in space which describes the evolution of a local phase variable $y$. Within a spatial domain $\Omega$, the latter is required to take values in $[-1,1]$ where, for $x \in \Omega, y(x)=1$ represents one of two phases and $y(x)=-1$ the other, respectively. For $-1<y(x)<1$ the composition is in a mixed state at $x \in \Omega$. Utilizing a chemical potential $w$ and assuming constant mobility (normalized to 1 ), the associated mathematical model reads

$$
\begin{array}{ccl}
y_{t}-\Delta w=0, & w+\gamma \Delta y \in \partial \Psi(y) & \text { in } \Omega, \\
\nabla w \cdot \vec{n}=0, & \nabla y \cdot \vec{n}=0 & \text { on } \partial \Omega
\end{array}
$$

${ }^{*}$ Received by the editors February 10, 2011; accepted for publication (in revised form) November 10, 2011; published electronically February 2, 2012. The authors gratefully acknowledge support by DFG Research Center MATHEON under project C28. M.H. further acknowledges support through FWF under START-program Y305-N18 "Interfaces and Free Boundaries," SFB F3204-N18 "Mathematical Optimization and Its Applications in Biomedical Sciences," and the DFG SPP 1253 "Optimization with Partial Differential Equations."

http://www.siam.org/journals/sicon/50-1/82415.html

${ }^{\dagger}$ Department of Mathematics, Humboldt-Universität zu Berlin, D-10099 Berlin, Germany and Department of Mathematics and Scientific Computing, Karl-Franzens-University of Graz, Graz, Austria (hint@math.hu-berlin.de).

${ }^{\ddagger}$ Department of Mathematics, Humboldt-Universität zu Berlin, D-10099 Berlin, Germany (dwegner@math.hu-berlin.de). 
with appropriate initial conditions. Here, $\gamma>0$ denotes a given parameter, $\Psi$ represents the homogeneous free energy density contained in the Ginzburg-Landau model, and $\partial \Psi$ stands for the generalized derivative from nonsmooth analysis [23]. Note that the latter is single-valued whenever $\Psi$ is differentiable at $y$. In this case $\partial \Psi(y)=\left\{\Psi^{\prime}(y)\right\}$ with $\Psi^{\prime}(y)$ the derivative of $\Psi$ at $y$.

Depending on the application context, different choices of $\Psi$ have been investigated in the literature. Typically, the various versions of $\Psi$ aim at confining the values of $y$ to $[-1,1]$ or $(-1,1)$. In this context, a widely studied choice is the double-well potential $[25,27,54]$. Another choice is of logarithmic form and goes back to Cahn and Hilliard's original work [21]; see also [2]. Logarithmic forms of the free energy density are also important in the Flory-Huggins solution theory of the thermodynamics of polymer solutions. While the double-well type free energy allows violations of $y(x) \in[-1,1]$, the logarithmic potential does not. Both choices, however, share certain differentiability properties such that $\partial \Psi$ becomes single-valued and the second equation in (1.1) becomes an equality with the derivative $\Psi^{\prime}$ on the right-hand side. On the other hand, in [55] Oono and Puri found that in the case of deep quenches of, e.g., binary alloys, the so-called double-obstacle potential

$$
\Psi(y)(x)= \begin{cases}\frac{1}{2}(1-y(x))^{2} & \text { if }|y(x)| \leq 1 \\ +\infty & \text { if }|y(x)|>1\end{cases}
$$

$x \in \Omega$, is better suited than the other free energy models mentioned above. A similar observation appears to be true in the case of polymeric membrane formation under rapid wall hardening. For this choice of the free energy, due to the nondifferentiability of the associated function $\Psi$ the second relation in the system (1.1) is indeed a variational inclusion or, equivalently, a variational inequality. For the resulting Cahn-Hilliard system, a comprehensive mathematical analysis can be found in $[17,18]$. Concerning numerical solvers we refer the reader to $[8,9,16,33,34,40]$ and the references therein.

In many applications, it might be interesting to influence the phase transition in such a way that a prespecified control goal is achieved. In this direction, feedback stabilization, as well as optimal control for the Cahn-Hilliard equation with a double-well type homogeneous free energy density, is studied theoretically in [62]. For a polynomial-type free energy density, in [60] a first-order optimality system is derived for minimizing a tracking-type objective subject to the associated CahnHilliard equation. With the goal of preventing spinodal decomposition in Fe-Al alloys, in [32] the Cahn-Hilliard system with double-well type free energy is controlled near a steady-state of the system. In some applications one might be interested in governing the Cahn-Hilliard system from an initial phase distribution (often a homogeneous mixture) $y_{0}$ to some desired phase pattern $y_{T}$ at a given (final) time $T$. For the Cahn-Hilliard system with a double-obstacle type homogeneous free energy density, such a problem formulation was mentioned by Garcke in [31]. For instance, in the context of polymeric membrane formation, $y_{T}$ may describe a desired porosity patter which implies filtration or other membrane qualities.

In this paper we pick up the latter perspective and study the minimization of an objective of the type

$J(y, u)=\frac{\mu_{1}}{2} \int_{0}^{T} \int_{\Omega}\left|y(x, t)-y_{\Omega}\right|^{2} d x d t+\frac{\mu_{2}}{2} \int_{\Omega}\left|y(x, T)-y_{T}\right|^{2} d x+\frac{1}{2} \int_{0}^{T} \int_{\Omega}|u(x, t)|^{2} d x d t$

subject to (1.1) with the double-obstacle homogeneous free energy density (1.2). Here, 
$\mu_{1}, \mu_{2} \geq 0$ are fixed and $y_{\Omega}$ and $y_{T}$ are given targets, respectively, and $u$ denotes the control variable. In this paper we consider control actions which enter through the right-hand side of the transport equation; i.e., we have

$$
y_{t}-\Delta w=u, \quad w+\gamma \Delta y \in \partial \Psi(y) .
$$

As noted above, the Cahn-Hilliard system with a double-obstacle type homogeneous free energy density admits an equivalent reformulation as a variational inequality such that the resulting minimization problem amounts to an optimal control problem for a parabolic variational inequality which is of fourth order in space.

Recently, in [41,42] optimal control problems for variational inequalities were linked to so-called mathematical programs with equilibrium constraints (MPECs). The latter problem class is well studied in finite dimensions; see, e.g., the monographs $[47,50,51,56]$ and the many references therein. It is well-known that MPECs are problematic from an optimization-theoretic point of view due to a generic lack of constraint qualification. This fact prevents the application of Karush-Kuhn-Tucker (KKT) type stationarity concepts for mathematical programs in Banach space [49,63]. In function space, the literature on MPECs is much scarcer and the work on the relation between finite and infinite dimensional versions of stationarity notions such as C-stationarity or strong $(S)$ stationarity is only at its beginning. Most problems in function space are formulated in terms of elliptic variational inequalities (see $[11,13,14$, $38,39,42,44,52]$ and the references therein, for instance). An account of parabolic-type variational inequalities can be found in the literature on mathematical finance $[3,4,43]$ and also in connection with the Stefan problem [11], as well as in the monograph [53] on the control of parabolic systems. Typically, the differential operator in these applications is of second order in space only, and to the best of our knowledge systems like the Cahn-Hilliard with double-obstacle potential are untreated in the literature.

In this paper our goal is to derive C-stationarity conditions for the minimization of (1.3) subject to (1.4) with the same boundary conditions as in (1.1) and with homogeneous free energy densities which cover the case of the double-obstacle free energy in (1.2). This will be achieved by a regularization process, which allows the application of classical KKT theory in Banach space [63] and the subsequent passage to the limit with respect to the regularization parameter. Concerning the control action, we study the case of distributed control as specified in (1.4) above. A further example for distributed control can be found, e.g., in [37], where a control problem for the Cahn-Hilliard equation with the double-well potential is studied.

Clearly, many applications require the coupling of the Cahn-Hilliard system with another system of partial differential equations describing the underlying physics. This could be the Navier-Stokes system [1] or linear or nonlinear elasticity [19,30,61], to mention only two. Coverage of such a fully coupled system, however, goes beyond the scope of the present work and rather justifies an independent study.

The remainder of the paper is organized as follows. In section 2 we introduce notation and provide existence and regularity results for solutions of parabolic differential inclusions. The optimal control problem is formulated in section 3 together with necessary assumptions on the involved operators. Then we study the existence of a solution of the original and the regularized MPEC as well as the behavior of solutions under a vanishing regularization parameter. Concerning the homogeneous free energy density we particularly highlight the distributed optimal control of the Cahn-Hilliard system with double-obstacle potential in section 4. For this particular case, for vanishing regularization parameter we obtain a function space version of C-stationarity conditions. 
2. Preliminaries. In this section we introduce the notation used throughout this work. We also provide an abstract existence result for evolution inclusions and results concerning the regularity of solutions.

2.1. Notation and conventions. For any real Banach space $X$ let us denote by $\|\cdot\|_{X}$ its norm, with $\langle., .\rangle_{X}: X^{*} \times X \rightarrow \mathbb{R}$ its dual pairing and the canonical injection of $X$ into its bidual space by $i_{X}: X \rightarrow X^{* *},\left\langle i_{X} x, x^{*}\right\rangle_{X^{*}}:=x^{*}(x)$. For $M \subset X$ we define a set $M^{+}:=\left\{x^{*} \in X^{*}:\left\langle x^{*}, m\right\rangle \geq 0\right.$ for all $\left.m \in M\right\} \subset X^{*}$. If $X$ is a Hilbert space, then $(. \mid .)_{X}: X \times X \rightarrow \mathbb{R}$ represents its inner product, and $J_{X}: X \rightarrow X^{*},\left\langle J_{X} x, y\right\rangle_{X}:=(x \mid y)_{X}$ denote the corresponding dual mapping. For two sets $M_{1} \subset M_{2}$ we denote by $I_{M_{1} \rightarrow M_{2}}: M_{1} \rightarrow M_{2}, I_{M_{1} \rightarrow M_{2}} x:=x$ the identity regarded as a mapping from $M_{1}$ into $M_{2}$. Finally, the convex indicator function $\imath_{M_{1}}: M_{2} \rightarrow \overline{\mathbb{R}}$ of $M_{1}$ is defined by

$$
\imath_{M_{1}}(x):= \begin{cases}0 & \text { if } x \in M_{1} \\ \infty & \text { otherwise }\end{cases}
$$

Let $\Omega$ be an open, bounded, and connected subset of $\mathbb{R}^{N}$ with smooth boundary, let $\vec{n}$ be its unit outer normal vector field, and let $\mathcal{T}=] 0, T$ [ be a bounded interval. For $n \in\{1, \ldots, 4\}$ we define the spaces $H, V_{n}$ by the following (for the definition of real-valued $L^{p}$-spaces, see, e.g., [5]; for vector-valued spaces, we refer the reader to $[29])$ :

$$
H:=\left\{u \in L^{2}(\Omega): \int_{\Omega} u=0\right\}
$$

$V_{n}:=\left\{u \in H^{n}(\Omega): \Delta^{k} u \in H,\left.\quad \nabla \Delta^{l} u \cdot \vec{n}\right|_{\partial \Omega}=0 \forall k, l \in \mathbb{N}_{0}\right.$ with $\left.2 k \leq n, 2 l+2 \leq n\right\}$.

In the above definition, $\Delta^{0}$ equals the identity and there is no condition on $\nabla \Delta^{l} u$ for $n=1$. The operators $L_{1}: V_{1} \rightarrow V_{1}^{*}, L_{n}: V_{n} \rightarrow V_{n-2}$ are given by

$$
\left\langle L_{1} u, v\right\rangle_{V_{1}}:=\int_{\Omega} \nabla u \cdot \nabla v, \quad L_{2} u:=-\Delta u, \quad L_{n}:=\left.L_{2}\right|_{V_{n}} .
$$

We equip $H$ with the $L^{2}$-inner product and $V_{1}, \ldots, V_{4}$ with the inner products

$$
(u \mid v)_{V_{1}}:=\left\langle L_{1} u, v\right\rangle_{V_{1}}, \quad(u \mid v)_{V_{n}}:=\left(L_{n} u \mid L_{n} v\right)_{V_{n-2}} .
$$

For $n \in\{1, \ldots, 4\}$ the corresponding time-dependent spaces are

$$
\mathcal{H}:=L^{2}(\mathcal{T} ; H), \quad \mathcal{V}_{n}:=L^{2}\left(\mathcal{T} ; V_{n}\right) .
$$

The spaces $H$ and $H^{*}$ will be identified via $J_{H}$. Furthermore, for $n \in\{3,4\}$ we use the abbreviations $I:=I_{V_{1} \rightarrow V_{1}}, \quad I_{1}:=I_{V_{1} \rightarrow V_{1}^{*}}, \quad I_{2}:=I_{V_{2} \rightarrow H}, \quad I_{n}:=I_{V_{n} \rightarrow V_{n-2}}$, and $I_{V_{1}}:=I_{V_{1} \rightarrow H}$.

For ease of notation we identify a multivalued operator $A: X \rightrightarrows Y$ with its graph $\operatorname{gph}(A) \subset X \times Y$, define its domain $D(A)$ as the set $\{x \in X: A x \neq \emptyset\}$, and denote the inverse and composition for $A \subset X \times Y, B \subset Y \times Z$ by

$$
A^{-1}:=\{(y, x):(x, y) \in A\}, \quad B A:=\left\{(x, z) \in X \times Z: A x \cap B^{-1} z \neq \emptyset\right\} .
$$

For multivalued operators from a Banach space $X$ into a Banach space $Y$ we denote by calligraphic letters the corresponding superposition operators mapping from $L^{2}(\mathcal{T} ; X)$ into $L^{2}(\mathcal{T} ; Y)$. For instance, for $A \subset X \times Y$ the operator $\mathcal{A}$ reads as

$$
\mathcal{A}:=\left\{(f, g) \in L^{2}(\mathcal{T} ; X) \times L^{2}(\mathcal{T} ; Y): g(t) \in A f(t) \text { for a.e. } t \in \mathcal{T}\right\} .
$$


Moreover, for a functional on $X$ denoted by a lowercase Greek letter, we use an uppercase Greek letter for its $L^{2}$-realization with respect to $\mathcal{T}$. If, for example, $\varphi$ : $X \rightarrow \overline{\mathbb{R}}$ is given, then $\Phi$ is defined as

$$
\Phi: L^{2}(\mathcal{T} ; X) \rightarrow \overline{\mathbb{R}}, \Phi(u):= \begin{cases}\int_{\Omega} \varphi \circ u & \text { if } \varphi \circ u \in L^{1}(\mathcal{T}) \\ \infty & \text { otherwise }\end{cases}
$$

For two Banach spaces $X$ and $Y$, we denote by $\mathcal{L}(X ; Y)$ the space of continuous linear operators from $X$ to $Y$. In the case $X=Y$, we also write $\mathcal{L}(X)$ instead of $\mathcal{L}(X ; X)$. Finally, assume that $E \in \mathcal{L}(X ; Y)$ is injective. Then the extension of $\varphi$ to $Y$ with respect to $E$ is given by

$$
\operatorname{Ext}(\varphi, X, Y, E): Y \rightarrow \overline{\mathbb{R}}, \quad \operatorname{Ext}(\varphi, X, Y, E)(y):= \begin{cases}\varphi\left(E^{-1} y\right) & \text { if } y \in E(X), \\ \infty & \text { otherwise. }\end{cases}
$$

Remark 2.1. 1. Since all the spaces $H, V_{1}, \ldots, V_{4}$ are reflexive, they possess the Radon-Nikodým property (cf. [26]). Hence, e.g., $\mathcal{V}_{1}^{*}$ can be identified with the space $L^{2}\left(\mathcal{T} ; V_{1}^{*}\right)$, and we shall do so without explicit use of the identification mapping. An analogous identification will be used for the other spaces.

2. The positive-definiteness of the inner products of $V_{1}, \ldots, V_{4}$ is a consequence of the Poincaré inequality and the fact that the only solution to the homogeneous Laplace equation with zero mean value and Neumann boundary conditions is the 0function. Moreover, it is not difficult to prove that these spaces are in fact Hilbert spaces.

3. Furthermore, the operator $L_{1}$ corresponds to the negative Laplacian with Neumann boundary conditions in its weak form as an operator from $V_{1}$ into $V_{1}^{*}$. By definition of the inner product of $V_{1}, L_{1}$ also coincides with the dual mapping of $V_{1}$, and all mappings $L_{1}, \ldots, L_{4}$ are unitary operators.

4. Since we identify the Hilbert space $H$ with its dual $H^{*}$ through $J_{H}$, and because $I_{V_{1}}: V_{1} \rightarrow H$ is linear, continuous, and has a dense range, the space $H \cong H^{*}$ becomes a subspace of $V_{1}^{*}$ (under the identification $I_{V_{1}}^{*}$ ). Additionally, Rellich's lemma implies that $I_{V_{1}}$ is a linear, compact mapping, and hence these properties transfer to $I_{V_{1}}^{*}$.

5. If $A: X \rightarrow Y$ is single-valued and defined on all of $X$, and if it is continuous and satisfies the growth condition

$$
\|A x\|_{Y} \leq C\left(1+\|x\|_{X}\right)
$$

for a constant $C>0$, then $\mathcal{A}$ is single-valued and defined on $L^{2}(\mathcal{T} ; X)$.

DeFinition 2.2. We define the following spaces that allow a weak (time-) derivative

$$
\begin{gathered}
\mathcal{W}_{1}:=\left\{u \in \mathcal{V}_{1}: u \in H^{1}\left(\mathcal{T} ; V_{1}^{*}\right)\right\}, \quad \mathcal{W}_{3}:=\left\{u \in \mathcal{V}_{3}: u \in H^{1}\left(\mathcal{T} ; V_{1}^{*}\right)\right\} \\
\mathcal{W}_{4}:=\left\{u \in \mathcal{V}_{4}: u \in H^{1}(\mathcal{T} ; H)\right\}
\end{gathered}
$$

We fix the following norms:

$$
\begin{gathered}
\|u\|_{\mathcal{W}_{1}}:=\left(\|u\|_{\mathcal{V}_{1}}^{2}+\left\|u^{\prime}\right\|_{\mathcal{V}_{1}^{*}}^{2}\right)^{1 / 2}, \quad\|u\|_{\mathcal{W}_{3}}:=\left(\|u\|_{\mathcal{V}_{3}}^{2}+\left\|u^{\prime}\right\|_{\mathcal{V}_{1}^{*}}^{2}\right)^{1 / 2} \\
\|u\|_{\mathcal{W}_{4}}:=\left(\|u\|_{\mathcal{V}_{4}}^{2}+\left\|u^{\prime}\right\|_{\mathcal{H}}^{2}\right)^{1 / 2}
\end{gathered}
$$

Most of the operators considered below are subdifferentials of convex functionals on various spaces. 
Definition 2.3. For every real Banach space $U$ and every functional $\varphi: U \rightarrow \overline{\mathbb{R}}$ we define a multivalued operator $\partial \varphi \subset U \times U^{*}$ by

$$
\partial \varphi:=\left\{\left(u, u^{*}\right) \in U \times U^{*}: u \in \operatorname{dom} \varphi \wedge \varphi(v)-\varphi(u) \geq\left\langle u^{*}, v-u\right\rangle_{U} \forall v \in U\right\} .
$$

If $U$ is a Hilbert space, we further introduce $\partial^{*} \varphi \subset U \times U$ :

$$
\partial^{*} \varphi:=J_{U}^{-1} \partial \varphi
$$

2.2. Existence results. Now we establish two properties of the extension of functionals that will be used below. The proofs of this lemma and of other basic results of this section are provided in the appendix.

Lemma 2.4. Let $X$ and $Y$ be two Banach spaces, let $E \in \mathcal{L}(X ; Y)$ be injective, and let $\varphi: X \rightarrow \overline{\mathbb{R}}$ a functional. Then we have

$$
\partial \operatorname{Ext}(\varphi, X, Y, E)=\left(E^{*}\right)^{-1} \partial \varphi E^{-1} .
$$

If, furthermore, $X$ is reflexive, $\varphi$ is proper, convex, and lower-semicontinuous and has bounded lower-level sets (i.e., $\left.\left.\varphi^{-1}(]-\infty, a\right]\right)$ is bounded in $X$ for every $\left.a \in \mathbb{R}\right)$, then $\operatorname{Ext}(\varphi, X, Y, E): Y \rightarrow \overline{\mathbb{R}}$ is proper, convex, and lower-semicontinuous as well.

The following theorem by Abels/Wilke [2, Theorem 3.1] is our central key to proving existence of solutions to evolution inclusions (cf. also [46]).

TheOREM 2.5. Assume that $W_{1}$ and $W_{0}$ are real, separable Hilbert spaces and that $E \in \mathcal{L}\left(W_{1} ; W_{0}\right)$ is injective with $E\left(W_{1}\right)$ dense in $W_{0}$. Let $\alpha>0$, let $\psi_{0}:=$ $\operatorname{Ext}\left(\alpha\|\cdot\|_{W_{1}}^{2}, W_{1}, W_{0}, E\right)$, and let $\psi: W_{0} \rightarrow \overline{\mathbb{R}}$ be a nonnegative, proper, convex, and lower-semicontinuous functional such that $\operatorname{dom} \psi \cap \operatorname{dom} \psi_{0} \neq \emptyset$. We set $\psi_{1}:=\psi_{0}+\psi$ and $R:=\partial^{*} \psi_{1}$. Furthermore, suppose that $S: W_{1} \rightarrow W_{0}$ is Lipschitz continuous. Then, for every $y_{0} \in D(R)$ and $f \in L^{2}\left(\mathcal{T} ; W_{0}\right)$ there exists a unique $y \in L^{\infty}\left(\mathcal{T} ; W_{1}\right)$ with $\mathcal{E} y \in H^{1}\left(\mathcal{T} ; W_{0}\right)$ such that

$$
\begin{aligned}
(\mathcal{E} y)^{\prime}+\mathcal{R E} y+\mathcal{S} y & \ni f, \\
(\mathcal{E} y)(0) & =y_{0} .
\end{aligned}
$$

Moreover, it holds that $\Psi_{1}(\mathcal{E} y) \in L^{\infty}(\mathcal{T})$.

Remark 2.6. The nonnegativity assumption on $\psi$ is not essential. Indeed, if $\psi: W_{0} \rightarrow \overline{\mathbb{R}}$ is proper, convex, and lower-semicontinuous, then it is bounded from below by an affine functional, i.e., $\psi(w) \geq\left(w_{0} \mid w\right)_{W_{0}}+r$ for some $w_{0} \in W_{0}, r \in \mathbb{R}$. Therefore, we can apply Theorem 2.5 to $\widetilde{\psi}(w):=\psi(w)-\left(w_{0} \mid w\right)_{W_{0}}-r$ and $\widetilde{S} w:=$ $S w+w_{0}$.

Next, we provide a reformulation of this theorem applied to our situation.

THEOREM 2.7. Suppose we are given a proper, convex, and lower-semicontinuous functional $\varphi: V_{1} \rightarrow \overline{\mathbb{R}}$, a Lipschitz mapping $B: V_{1} \rightarrow V_{1}, y_{0} \in D\left(\partial \operatorname{Ext}\left(\varphi, V_{1}, V_{1}^{*}, I_{1}\right)\right)$, and $f \in \mathcal{V}_{1}^{*}$. Then there exists a unique $y \in \mathcal{W}_{1} \cap L^{\infty}\left(\mathcal{T} ; V_{1}\right)$ with

$$
\begin{aligned}
\left(\mathcal{I}_{1} y\right)^{\prime}+\mathcal{L}_{1} w & =f, \\
\mathcal{L}_{1} y+(\partial \Phi)(y)+\mathcal{I}_{1} \mathcal{B} y & \ni w, \\
\left(\mathcal{I}_{1} y\right)(0) & =y_{0} .
\end{aligned}
$$

Additionally, y satisfies $\varphi(y)+\frac{1}{2}\|y\|_{V_{1}}^{2} \in L^{\infty}(\mathcal{T})$.

Proof. We define $\varphi_{0}, \varphi_{1}: V_{1} \rightarrow \overline{\mathbb{R}}$ by $\varphi_{0}:=\frac{1}{2}\|.\|_{V_{1}}^{2}, \varphi_{1}:=\varphi_{0}+\varphi$, and $A:=\partial \varphi$ and apply Theorem 2.5 to the following setting:

$$
W_{1}:=V_{1}, \quad W_{0}:=V_{1}^{*}, \quad E:=I_{1}, \quad \alpha:=\frac{1}{2}, \quad R:=\partial^{*} \Psi_{1}, \quad S:=L_{1} B,
$$


where $\psi, \psi_{0}$, and $\psi_{1}$ denote the extensions of $\varphi, \varphi_{0}$, and $\varphi_{1}$, to $V_{1}^{*}$ with respect to $I_{1}$. From Lemma 2.4 it follows that $\psi$ is proper, convex, and lower-semicontinuous. Hence, there exists a unique $y \in L^{\infty}\left(\mathcal{T} ; V_{1}\right)$ with $\mathcal{I}_{1} y \in H^{1}\left(\mathcal{T} ; V_{1}^{*}\right), \Psi_{1}\left(\mathcal{I}_{1} y\right) \in L^{\infty}(\mathcal{T})$ and

$$
\left(\mathcal{I}_{1} y\right)^{\prime}+\mathcal{R} \mathcal{I}_{1} y+\mathcal{S} y \ni f, \quad\left(\mathcal{I}_{1} y\right)(0)=y_{0} .
$$

In order to calculate $\mathcal{R}$ we use two basic facts from functional analysis:

(1) For every Hilbert space $W$ it holds that $i_{W}=J_{W^{*}} J_{W}, J_{W^{*}}^{-1}=J_{W}^{*}$.

(2) For Banach spaces $X, Y$ and $F \in \mathcal{L}(X ; Y)$ we have $F^{* *} i_{X}=i_{Y} F$.

Thus, $J_{H}^{*} i_{H}=J_{H^{*}}^{-1} J_{H^{*}} J_{H}=J_{H}$, and from $I_{1}=I_{V_{1}}^{*} J_{H} I_{V_{1}}$ it follows that

$$
I_{1}^{*}=I_{V_{1}}^{*} J_{H}^{*} I_{V_{1}}^{* *}=I_{V_{1}}^{*} J_{H}^{*} i_{H} I_{V_{1}} i_{V_{1}}^{-1}=I_{1} i_{V_{1}}^{-1}=I_{1} J_{V_{1}}^{-1} J_{V_{1}^{*}}^{-1} .
$$

Hence, Lemma 2.4 yields

$R=J_{V_{1}^{*}}^{-1} \partial \operatorname{Ext}\left(\varphi_{1}, V_{1}, V_{1}^{*}, I_{1}\right)=J_{V_{1}^{*}}^{-1}\left(I_{1}^{*}\right)^{-1} \partial \varphi_{1} I_{1}^{-1}=J_{V_{1}} I_{1}^{-1} \partial \varphi_{1} I_{1}^{-1}=L_{1} I_{1}^{-1} \partial \varphi_{1} I_{1}^{-1}$,

and since $\varphi_{1}=\varphi_{0}+\varphi$ with $\varphi_{0}$ being continuous on $V_{1}$ and therefore $\partial \varphi_{1}=\partial \varphi_{0}+\partial \varphi=$ $L_{1}+A$, it follows that

$$
R I_{1}=L_{1} I_{1}^{-1} \partial \varphi_{1}=L_{1} I_{1}^{-1}\left(L_{1}+A\right) .
$$

Consequently, (2.4) is equivalent to $\left(\mathcal{I}_{1} y\right)^{\prime}+\mathcal{L}_{1} \mathcal{I}_{1}^{-1}\left(\mathcal{L}_{1}+\mathcal{A}+\mathcal{I}_{1} \mathcal{B}\right) y \ni f$. Defining $w:=\mathcal{L}_{1}^{-1}\left(f-\left(\mathcal{I}_{1} y\right)^{\prime}\right)$, this can be rewritten as

$$
\left(\mathcal{I}_{1} y\right)^{\prime}+\mathcal{L}_{1} w=f, \quad\left(\mathcal{L}_{1}+\mathcal{A}+\mathcal{I}_{1} \mathcal{B}\right) y \ni \mathcal{I}_{1} w,
$$

which completes the proof.

2.3. Regularity results. The following proposition provides some basic properties of the interplay between $I_{1}$ and $L_{1}$.

Proposition 2.8. The following commutation rules hold true:

$$
I_{1} L_{3}=L_{1} I_{3} \quad \text { and } \quad I_{3}^{*} L_{1}=L_{3}^{*} I_{1} .
$$

Moreover, $L_{3}: V_{3} \rightarrow V_{1}, L_{1}: V_{1} \rightarrow V_{1}^{*}$, and $L_{3}^{*}: V_{1}^{*} \rightarrow V_{3}^{*}$ are unitary operators, and the mappings $L_{1}^{-1} I_{1} \in \mathcal{L}\left(V_{1}\right)$ and $\left(L_{3}^{*}\right)^{-1} I_{3}^{*}$ are positive, symmetric, injective, and compact. If $U_{1} \subset V_{1}$ and $U_{1}^{*} \subset V_{1}^{*}$ are closed subspaces such that $L_{1}^{-1} I_{1}\left(U_{1}\right)=U_{1}$ and $\left(L_{3}^{*}\right)^{-1} I_{3}^{*}\left(U_{1}^{*}\right)=U_{1}^{*}$ are satisfied, then it holds that

$$
\begin{gathered}
I_{1} P_{V_{1} \rightarrow U_{1}}=P_{V_{1}^{*} \rightarrow I_{1}\left(U_{1}\right)} I_{1}, I_{3}^{*} P_{V_{1}^{*} \rightarrow U_{1}^{*}}=P_{V_{3}^{*} \rightarrow I_{3}^{*}\left(U_{1}^{*}\right)} I_{3}^{*}, \\
L_{1} P_{V_{1} \rightarrow U_{1}}=P_{V_{1}^{*} \rightarrow I_{1}\left(U_{1}\right)} L_{1}, L_{3}^{*} P_{V_{1}^{*} \rightarrow U_{1}^{*}}=P_{V_{3}^{*} \rightarrow I_{3}^{*}\left(U_{1}^{*}\right)} L_{3}^{*}
\end{gathered}
$$

with $P_{X \rightarrow Y}$ denoting the othogonal projection of $X$ onto the closed subspace $Y \subset X$.

Furthermore, the following generalization of the integration-by-parts formula to functions admitting time-derivatives in different spaces is needed.

Proposition 2.9. Assume that $y \in \mathcal{V}_{3}, v \in \mathcal{V}_{1}$ with $y \in H^{1}\left(\mathcal{T} ; V_{1}^{*}\right)$ and $v \in H^{1}\left(\mathcal{T} ; V_{3}^{*}\right)$. Then $y$ and $v$ possess representatives in $C\left(\mathcal{T} ; V_{1}\right)$ and $C\left(\mathcal{T} ; V_{1}^{*}\right)$, respectively, which can be continuously extended to $\overline{\mathcal{T}}$. Moreover, in this sense it holds that

$$
\left\langle y^{\prime}, v\right\rangle_{\mathcal{V}_{1}}+\left\langle v^{\prime}, y\right\rangle_{\mathcal{V}_{3}}=\langle v(T), y(T)\rangle_{V_{1}}-\langle v(0), y(0)\rangle_{V_{1}}
$$


Next, we state a regularity result for the time-dependent bi-Laplace equation ensuring higher space regularity.

THEOREM 2.10. Suppose that $y \in \mathcal{V}_{1}$ and $f \in \mathcal{V}_{1}^{*}$ satisfy $y \in H^{1}\left(\mathcal{T} ; V_{3}^{*}\right), y(0) \in V_{1}$ together with $y^{\prime}+\mathcal{L}_{3}^{*} \mathcal{L}_{1} y=f$ in $\mathcal{V}_{3}^{*}$. Then $y \in \mathcal{V}_{3}, y \in H^{1}\left(\mathcal{T} ; V_{1}^{*}\right)$ and it holds that

$$
y^{\prime}+\mathcal{L}_{1} \mathcal{L}_{3} y=f \text { in } \mathcal{V}_{1}^{*} .
$$

Remark 2.11. If we assume that $f \in \mathcal{H}$ and $y(0) \in V_{2}$ are satisfied, then $y \in$ $\mathcal{V}_{4}, y \in H^{1}(\mathcal{T} ; H)$, and $y^{\prime}+\mathcal{L}_{2} \mathcal{L}_{4} y=f \in \mathcal{H}$.

In order to derive an optimality system later on, the continuous Fréchet differentiability of some superposition operators acting on integrable functions with values in Sobolev spaces will be needed.

Proposition 2.12. Let $\Omega \subset \mathbb{R}^{N}$ be an open, bounded set with smooth boundary, and let $\gamma: \mathbb{R} \rightarrow \mathbb{R}$ be twice continuously differentiable with bounded first and second derivatives. Let $\mathcal{A}$ denote the superposition operator

$$
\mathcal{A}: L^{s_{1}}\left(\mathcal{T} ; W^{1, s_{2}}(\Omega)\right) \rightarrow L^{2}\left(\mathcal{T} ; H^{1}(\Omega)\right), \quad(\mathcal{A} y)(t, x):=\gamma(y(t, x)) .
$$

If $s_{1} \geq 4$ and $s_{2}>2$ with $s_{2} \geq \frac{4 N}{N+2}$, then the operator $\mathcal{A}$ is continuously Fréchet differentiable, and its derivative $\mathcal{A}^{\prime}: L^{s_{1}}\left(\mathcal{T} ; W^{1, s_{2}}(\Omega)\right) \rightarrow \mathcal{L}\left(L^{s_{1}}\left(\mathcal{T} ; W^{1, s_{2}}(\Omega)\right) ; L^{2}\left(\mathcal{T} ; H^{1}(\Omega)\right)\right)$ is given by

$$
\left(\mathcal{A}^{\prime}(y ; r)\right)(t, x)=\gamma^{\prime}(y(t, x)) r(t, x) .
$$

3. Optimal control for the Cahn-Hilliard system. Now, we turn our attention to a problem of optimal control of the Cahn-Hilliard system. For theoretical as well as numerical purposes, the original problem is approximated by a suitable sequence of associated auxiliary problems which are easier to handle from an optimization-theoretic point of view. We then derive an optimality system for the auxiliary problems which permits us to pass to the limit. This results in first-order optimality conditions of C-stationarity type for the original problem.

In addition to our earlier notation and assumptions we invoke the following assumption.

Assumption 3.1. For $\alpha_{0}>0$ and $\left.\left.\Lambda:=\right] 0, \alpha_{0}\right], \bar{\Lambda}:=\left[0, \alpha_{0}\right]$ let $\left(\varphi_{\alpha}\right)_{\alpha \in \bar{\Lambda}}$ be proper, convex, and lower-semicontinuous functionals on $V_{1}$ with values in $\overline{\mathbb{R}}$, and let $\mathcal{C} \subset \mathcal{H}$ be closed, convex, and nonempty. We fix $\eta \leq 0$ and define $\psi:=-\frac{\eta}{2}\left\|I_{V_{1}}(.)\right\|_{H}^{2}$, $\varphi:=\varphi_{0}, \theta_{\alpha}:=\frac{1}{2}\|\cdot\|_{V_{1}}^{2}+\varphi_{\alpha}-\psi: V_{1} \rightarrow \overline{\mathbb{R}}$ and the operators $A_{\alpha}:=\partial \varphi_{\alpha}, A:=\partial \varphi$ for $\alpha \in \bar{\Lambda}$. We suppose the following:

(i) $\left(A_{\alpha}\right)_{\alpha \in \Lambda}$ are single-valued operators from $V_{1}$ into $V_{1}^{*}$.

(ii) There exists some constant $c_{\theta}>0$ such that the functionals $\left(\theta_{\alpha}-c_{\theta}\|\cdot\|_{V_{1}}^{2}\right)_{\alpha \in \bar{\Lambda}}$ are bounded from below by some common constant.

(iii) The restrictions of the superposition operators $\left(\mathcal{A}_{\alpha}\right)_{\alpha \in \Lambda}$ of $\left(A_{\alpha}\right)_{\alpha \in \Lambda}$ to the subspace $\mathcal{W}_{1} \subset \mathcal{V}_{1}$ attain only values in $\mathcal{V}_{1} \subset \mathcal{V}_{1}^{*}$. We define for $\alpha \in \Lambda$

$$
\widehat{\mathcal{A}}_{\alpha}: \mathcal{W}_{1} \rightarrow \mathcal{V}_{1}, \quad \widehat{\mathcal{A}}_{\alpha}:=\mathcal{A}_{\alpha} .
$$

(iv) For every $\alpha \in \Lambda$, the operator $\left(\widehat{\mathcal{A}}_{\alpha}\right)_{\alpha \in \Lambda}$ is continuously Fréchet differentiable from $\mathcal{W}_{3}$ into $\mathcal{V}_{1}$. In addition, for every $y \in \mathcal{W}_{4}$ its derivative $D \widehat{\mathcal{A}}_{\alpha}(y)$ can be continuously extended to a linear, bounded operator $\overline{D \mathcal{A}_{\alpha}}(y)$ from $\mathcal{V}_{1}$ into itself.

(v) For all $\alpha \in \Lambda$ and $y \in \mathcal{W}_{3}$ it holds that $\left\langle\mathcal{L}_{1} y, \widehat{\mathcal{A}}_{\alpha} y\right\rangle_{\mathcal{V}_{1}} \geq 0$. 
(vi) Moreover, if $\left(\alpha_{n}\right)$ and $\left(y_{n}\right)$ are sequences in $\Lambda$ and $\mathcal{W}_{1}$ such that $\alpha_{n} \rightarrow 0$, $y_{n} \rightarrow y$ in $\mathcal{W}_{1}, y_{n} \rightarrow y$ in $\mathcal{H}$, and $\alpha \in \Lambda$, then

(1) $\mathcal{A}_{\alpha} y_{n} \rightarrow \mathcal{A}_{\alpha} y$ in $\mathcal{V}_{1}^{*}$,

(2) if $\widehat{\mathcal{A}}_{\alpha_{n}} y_{n} \rightarrow h$ in $\mathcal{H}$, then $(y, h) \in \mathcal{A}$.

We fix $y_{\Omega} \in \mathcal{H}, y_{T} \in V_{1}, \mu_{1}, \mu_{2} \geq 0$ and define the functional $J: \mathcal{W}_{1} \times \mathcal{H} \rightarrow \mathbb{R}$ by

$$
J(y, u):=\frac{1}{2}\left(\mu_{1}\left\|y-y_{\Omega}\right\|_{\mathcal{H}}^{2}+\mu_{2}\left\|y(T)-y_{T}\right\|_{H}^{2}+\|u\|_{\mathcal{H}}^{2}\right) .
$$

Finally, let $y_{0} \in V_{1}$ be given such that $y_{0} \in D\left(\partial \operatorname{Ext}\left(\varphi_{\alpha}, V_{1}, V_{1}^{*}, I_{1}\right)\right)$ for $\alpha \in \bar{\Lambda}$ and $\sup \left\{\theta_{\alpha}\left(y_{0}\right): \alpha \in \bar{\Lambda}\right\}=: C_{\theta}<\infty$.

Throughout the rest of the paper, we refer to $y$ as the state and $u$ as the control variable, respectively.

For given $u \in \mathcal{H}$ and $\alpha \in \bar{\Lambda}$ consider the problems of finding $y \in \mathcal{W}_{1}$ such that there is a $w \in \mathcal{V}_{1}$ with

$$
y^{\prime}+\mathcal{L}_{1} w=u, \quad w \in\left(\mathcal{L}_{1}+\mathcal{A}_{\alpha}+\eta \mathcal{I}_{1}\right) y, \quad y(0)=y_{0},
$$

and $(\mathrm{Q}):=\left(\mathrm{Q}_{0}\right)$. The following theorem shows that the problem $\left(\mathrm{Q}_{\alpha}\right)$ admits a unique solution.

Remark 3.2. In section 4 we focus on the special case where $\varphi$ is the convex indicator function of the set $\left\{v \in V_{1}: v(x) \in[a, b]\right.$ a.e. on $\left.\Omega\right\}$ for $a, b \in \mathbb{R}, a<b$, and $\varphi_{\alpha}$ for $\alpha>0$ is some suitable mollified version of its Moreau-Yosida approximation. Note that under these conditions and for $\alpha>0,\left(\mathrm{Q}_{\alpha}\right)$ represents a regularized version of the Cahn-Hilliard system with double-obstacle homogeneous free energy density. The regularization acts on the indicator function involved in the potential. For $\alpha=0$ we arrive at the original Cahn-Hilliard system with double-obstacle potential.

Theorem 3.3. For every $\alpha \in \bar{\Lambda}$ and every right-hand side $u \in \mathcal{H}$ the problem $\left(\mathrm{Q}_{\alpha}\right)$ has a unique solution $y \in \mathcal{W}_{1}$. If $\alpha \in \Lambda$, the solution satisfies $y \in \mathcal{W}_{3}$, and with $w:=\mathcal{L}_{1}^{-1}\left(u-y^{\prime}\right)$, we have

$$
y^{\prime}+\mathcal{L}_{1} w=u, \quad w=\left(\mathcal{L}_{3}+\widehat{\mathcal{A}}_{\alpha}+\eta \mathcal{I}\right) y, \quad y(0)=y_{0},
$$

Proof. The existence and uniqueness of a solution are direct consequences of Theorem 2.7. For given $\alpha \in \Lambda$, let $y \in \mathcal{W}_{1}$ be the solution to $\left(\mathrm{Q}_{\alpha}\right)$ and $w:=$ $\mathcal{L}_{1}^{-1}\left(u-y^{\prime}\right)$. Then

$$
\mathcal{I}_{3}^{*}\left(y^{\prime}+\mathcal{L}_{1} w\right)=y^{\prime}+\mathcal{L}_{3}^{*} w=y^{\prime}+\mathcal{L}_{3}^{*}\left(\mathcal{L}_{1} y+\mathcal{A}_{\alpha} y+\eta \mathcal{I}_{1} y\right)
$$

and since $\mathcal{A}_{\alpha} y, \eta \mathcal{I}_{1} y \in \mathcal{V}_{1}$, it follows that $\mathcal{L}_{3}^{*}\left(\mathcal{A}_{\alpha} y+\eta \mathcal{I}_{1} y\right)=\mathcal{L}_{3}^{*} \mathcal{I}_{1} \mathcal{I}_{1}^{-1}\left(\mathcal{A}_{\alpha} y+\eta \mathcal{I}_{1} y\right)=$ $\mathcal{L}_{1} \mathcal{I}_{1}^{-1}\left(\mathcal{A}_{\alpha} y+\eta \mathcal{I}_{1} y\right) \in \mathcal{V}_{1}^{*}$. Consequently, Theorem 2.10 implies the assertion.

Remark 3.4. Theorem 3.3 shows that the original problem (Q) admits a solution $y \in \mathcal{W}_{1}$ for every $u \in \mathcal{H}$. Furthermore, the solutions $y$ of the regularized problems $\left(\mathrm{Q}_{\alpha}\right)$ belong to $\mathcal{W}_{3}$ for all $\alpha \in \Lambda$. This holds true even for all $u \in \mathcal{V}_{1}^{*}$ and will be used to prove the existence of Lagrange multipliers for the optimal control problem $\left(\mathrm{P}_{\alpha}\right)$ below. For $u \in \mathcal{H}$ we can use Remark 2.11 and obtain $y \in \mathcal{W}_{4}$ for $\alpha>0$. This higher regularity will be needed only to show that in the linearization of $\left(\mathrm{Q}_{\alpha}\right)$ the derivative $D \widehat{\mathcal{A}}_{\alpha}(y)$ for $y \in \mathcal{W}_{4}$ extends continuously to a operator in $\mathcal{L}\left(\mathcal{V}_{1} ; \mathcal{V}_{1}\right)$ (which, in general, does not hold for $y \in \mathcal{W}_{3}$ ).

Definition 3.5. For given $u \in \mathcal{H}$, let $S_{\alpha} u \in \mathcal{W}_{1}$ denote the solution of $\left(\mathrm{Q}_{\alpha}\right)$ given by Theorem 3.3 . 
3.1. Regularized optimal control problems. In order to derive an optimality system for problem (P) below, the nonsmooth potential $\varphi$ is replaced by $\varphi_{\alpha}$ leading to the family of smooth optimization problems $\left(\mathrm{P}_{\alpha}\right)$, which we study next.

For $\alpha \in \bar{\Lambda}$ and with $(\mathrm{P}):=\left(\mathrm{P}_{0}\right)$ we consider

$$
\left(\mathrm{P}_{\alpha}\right) \quad \inf \left\{J(y, u):(y, u) \in \mathcal{W}_{1} \times \mathcal{C}, y=S_{\alpha} u\right\} .
$$

For studying $\alpha \rightarrow 0$ in $\left(\mathrm{P}_{\alpha}\right)$ we need the energy estimate for a solution to problem $\left(\mathrm{Q}_{\alpha}\right)$ as given in the next lemma.

Lemma 3.6. Let $u \in \mathcal{H}$ and $\alpha \in \bar{\Lambda}$ be given. For the corresponding solution $y:=S_{\alpha} u \in \mathcal{W}_{1}$ to problem $\left(\mathrm{Q}_{\alpha}\right)$ and $w:=\mathcal{L}_{1}^{-1}\left(u-y^{\prime}\right)$ it holds that $\theta_{\alpha} \circ y$ admits an absolutely continuous representative on $\overline{\mathcal{T}}$, and in this sense for $t \in \overline{\mathcal{T}}$ and $\mathcal{T}^{\prime}:=[0, t]$ we have

$$
\left(\theta_{\alpha} \circ y\right)(t)+\left\|\chi_{\mathcal{T}^{\prime}} w\right\|_{\mathcal{V}_{1}}^{2}=\left(\theta_{\alpha} \circ y\right)(0)+\left\langle u, \chi_{\mathcal{T}^{\prime}} w\right\rangle_{\mathcal{V}_{1}} .
$$

In particular, it holds that $\left\|\theta_{\alpha} \circ y\right\|_{L^{\infty}(\mathcal{T})}+\|w\|_{\mathcal{V}_{1}}^{2} \leq\left(\theta_{\alpha} \circ y\right)(0)+\|u\|_{\mathcal{V}_{1}^{*}}\|w\|_{\mathcal{V}_{1}}$.

Proof. The chain rule (cf. Proposition 4.2 in [24]) implies for the convex functionals $\sigma=\theta_{\alpha}+\psi$ and $\sigma=\psi$ that $\sigma \circ y$ is absolutely continuous on $\overline{\mathcal{T}}$ and for every $v \in \mathcal{V}_{1}$ such that $v(t) \in \partial \sigma(y(t))$ for almost all $t \in \overline{\mathcal{T}}$, and with $\mathcal{T}^{\prime}:=[0, t]$ it holds that

$$
(\sigma \circ y)(t)=\int_{\mathcal{T}^{\prime}} \frac{d}{d t}(\sigma \circ y) d t+(\sigma \circ y)(0)=\left\langle y^{\prime}, \chi_{\mathcal{T}^{\prime}} v\right\rangle_{\mathcal{V}_{1}}+(\sigma \circ y)(0) .
$$

Using $w \in\left(\mathcal{L}_{1}+\mathcal{A}_{\alpha}+\eta \mathcal{I}_{1}\right) y=\left(\partial\left(\Theta_{\alpha}+\Psi\right)-\partial \Psi\right) y$, we obtain

$$
\begin{aligned}
\left(\theta_{\alpha} \circ y\right)(t) & =\left\langle y^{\prime}, \chi_{\mathcal{T}}, w\right\rangle_{\mathcal{V}_{1}}+\left(\theta_{\alpha} \circ y\right)(0) \\
& =\left\langle u-\mathcal{L}_{1} w, \chi_{\mathcal{T}^{\prime}} w\right\rangle_{\mathcal{V}_{1}}+\left(\theta_{\alpha} \circ y\right)(0) \\
& =-\left\|\chi_{\mathcal{T}^{\prime}} w\right\|_{\mathcal{V}_{1}}^{2}+\left\langle u, \chi_{\mathcal{T}}, w\right\rangle_{\mathcal{V}_{1}}+\left(\theta_{\alpha} \circ y\right)(0),
\end{aligned}
$$

which yields the desired assertions.

Corollary 3.7. Let $\left(\alpha_{n}\right)$ and $\left(u_{n}\right)$ be sequences in $\bar{\Lambda}$ and in $\mathcal{H}$, respectively, such that $\left(u_{n}\right)$ is bounded in $\mathcal{V}_{1}^{*}$. By $\left(y_{n}\right)$ we denote the sequence of corresponding solution $S_{\alpha_{n}} u_{n}$ to the problem $\left(\mathrm{Q}_{\alpha_{n}}\right)$ and $w_{n}:=\mathcal{L}_{1}^{-1}\left(u_{n}-y_{n}^{\prime}\right)$. Then $\left(y_{n}\right)$ is bounded in $\mathcal{W}_{1}$ and $L^{\infty}\left(\mathcal{T} ; V_{1}\right)$, and $\left(w_{n}\right)$ is bounded in $\mathcal{V}_{1}$. If $\alpha_{n} \in \Lambda$ for all $n$, then $\widehat{\mathcal{A}}_{\alpha_{n}} y_{n}$ is bounded in $\mathcal{H}$.

Proof. From the energy estimate of Lemma 3.6 it follows that $\left(w_{n}\right)$ is bounded in $\mathcal{V}_{1}$. Hence, $y_{n}^{\prime}=u_{n}-\mathcal{L}_{1} w_{n}$ is bounded in $\mathcal{V}_{1}^{*}$. Moreover, the energy estimate implies that $\left(y_{n}\right)$ is bounded in $L^{\infty}\left(\mathcal{T} ; V_{1}\right)$ since $\theta_{\alpha} \geq c_{\theta}\|.\|_{V_{1}}^{2}-c$ for all $\alpha \in \bar{\Lambda}$ and some $c \in \mathbb{R}$. Hence, $\left(y_{n}\right)$ is also bounded in $\mathcal{W}_{1}$. If $\alpha_{n} \in \Lambda$ for all $n$, then we obtain from $w_{n}=\left(\mathcal{L}_{1}+\widehat{\mathcal{A}}_{\alpha_{n}}+\eta \mathcal{I}_{1}\right) y_{n}$, and with the help of Assumption 3.1(iv)-(v), that

$$
\begin{aligned}
\left\|\widehat{\mathcal{A}}_{\alpha_{n}} y_{n}\right\|_{\mathcal{H}}^{2} & =\left\langle\widehat{\mathcal{A}}_{\alpha_{n}} y_{n}, \widehat{\mathcal{A}}_{\alpha_{n}} y_{n}\right\rangle_{\mathcal{V}_{1}}=\left\langle w_{n}-\left(\mathcal{L}_{1}+\eta \mathcal{I}_{1}\right) y_{n}, \widehat{\mathcal{A}}_{\alpha_{n}} y_{n}\right\rangle_{\mathcal{V}_{1}} \\
& \leq\left(w_{n}-\eta \mathcal{I}_{1} y_{n} \mid \widehat{\mathcal{A}}_{\alpha_{n}} y_{n}\right)_{\mathcal{H}} \leq C\left\|\widehat{\mathcal{A}}_{\alpha_{n}} y_{n}\right\|_{\mathcal{H}} .
\end{aligned}
$$

This finishes the proof.

With these a priori estimates we are able to prove convergence (consistence) results and the existence of minimizers for $\left(\mathrm{P}_{\alpha}\right)$. 
Proposition 3.8. Let $\left(\alpha_{n}\right)$ and $\left(u_{n}\right)$ be sequences in $\bar{\Lambda}$, respectively, $\mathcal{H}$, with $\alpha_{n} \rightarrow 0$ and $u_{n} \rightarrow u$ in $\mathcal{V}_{1}^{*}$ for some $u \in \mathcal{H}$. Then there exist subsequences (denoted by the index $m$ ) such that

$$
S_{\alpha_{m}} u_{m} \rightarrow \text { Su } \quad \text { in } \mathcal{W}_{1}, S_{\alpha_{m}} u_{m} \rightarrow \text { Su in } \mathcal{V}_{1}
$$

Proof. From Corollary 3.7 we already know that $y_{n}:=S_{\alpha_{n}} u_{n}$ is bounded in $\mathcal{W}_{1}$ and $w_{n}:=\mathcal{L}_{1}^{-1}\left(u_{n}-y_{n}^{\prime}\right)$ in $\mathcal{V}_{1}$. Due to the compactness of $\mathcal{I}_{1}: \mathcal{W}_{1} \rightarrow \mathcal{H}$ and with $a_{n}:=w_{n}-\left(\mathcal{L}_{1}+\eta \mathcal{I}_{1}\right) y_{n} \in \mathcal{A}_{\alpha_{n}} y_{n}$ we have that

$$
\begin{array}{cl}
\left(y_{m}, w_{m}, a_{m}\right) \rightarrow(y, w, a) & \text { in } \mathcal{W}_{1} \times \mathcal{V}_{1} \times \mathcal{V}_{1}^{*}, \\
y_{m} \rightarrow y & \text { in } \mathcal{H}
\end{array}
$$

for some $y \in \mathcal{W}_{1}, w \in \mathcal{V}_{1}$, and $a \in \mathcal{V}_{1}^{*}$ along a subsequence $\alpha_{m}$ of $\alpha_{n}$. Moreover, since $w_{n}=\mathcal{L}_{1} y_{n}+a_{n}+\eta y_{n}$, it follows that $w=\mathcal{L}_{1} y+a+\eta y$ as well as

$$
\begin{aligned}
\left\|y_{m}-y\right\|_{\mathcal{V}_{1}}^{2} & =\left\langle\mathcal{L}_{1}\left(y_{m}-y\right), y_{m}-y\right\rangle_{\mathcal{V}_{1}} \\
& =\left(w_{m}-w \mid y_{m}-y\right)_{\mathcal{H}}-\left\langle a_{m}-a, y_{m}-y\right\rangle_{\mathcal{V}_{1}}-\left\langle\eta y_{m}-\eta y, y_{m}-y\right\rangle_{\mathcal{V}_{1}}
\end{aligned}
$$

for $m \in \mathbb{N}$. The strong convergence of $y_{m}$ in $\mathcal{H}$, respectively, $\mathcal{V}_{1}^{*}$, shows that the first and the last term on the right-hand side tend to zero. In the case that $\alpha_{m}=0$ for infinitely many $m$, for these indices it holds that $\left\langle a_{m}-a, y_{m}-y\right\rangle_{\mathcal{V}_{1}} \geq 0$ by the monotonicity of $\mathcal{A}$, and hence $y_{m} \rightarrow y$ in $\mathcal{V}_{1}$. Thus, from Proposition 2.5 of [20] we obtain that $(y, a) \in \mathcal{A}$.

Otherwise, we may assume the $\alpha_{m}>0$ for all $m$ and that $\widehat{\mathcal{A}}_{\alpha_{m}} y_{m}$ converges weakly in $\mathcal{H}$ to an $a_{1} \in \mathcal{H}$ by Corollary 3.7. This implies $\left\langle a_{m}-a, y_{m}-y\right\rangle_{\mathcal{V}_{1}}=$ $\left(a_{m}-a \mid y_{m}-y\right)_{\mathcal{H}} \rightarrow 0$. Consequently, it holds that $y_{m} \rightarrow y$ in $\mathcal{V}_{1}$ and that $a=a_{1}$ and $\left(y, a_{1}\right) \in \mathcal{A}$ by Assumption 3.1(vi).

Proposition 3.9. For every $\alpha \in \bar{\Lambda}$ the problem $\left(\mathrm{P}_{\alpha}\right)$ admits a minimizer $(y, u) \in$ $\mathcal{W}_{1} \times \mathcal{C}$.

Proof. Although the proof technique is standard, we provide the proof for the sake of keeping the paper self-contained. Let $\alpha \in \bar{\Lambda}$ be given, and let $\left(S_{\alpha} u_{n}, u_{n}\right)$ be an infimizing sequence for problem $\left(\mathrm{P}_{\alpha}\right)$. We set $y_{n}:=S_{\alpha} u_{n} \in \mathcal{W}_{1}$ and $w_{n}:=$ $\mathcal{L}_{1}^{-1}\left(u_{n}-y_{n}^{\prime}\right)$. The coercivity of $J$ yields that $\left(u_{n}\right)$ is bounded in $\mathcal{H}$. With the help of Corollary 3.7 we may pass to subsequences (denoted by the index $m$ ) such that

$$
\begin{array}{cl}
\left(y_{m}, u_{m}\right) \rightarrow(y, u) & \text { in } \mathcal{W}_{1} \times \mathcal{H}, \\
y_{m} \rightarrow y & \text { in } \mathcal{H} .
\end{array}
$$

The continuity properties of $\mathcal{A}_{\alpha}$ given in Assumption 3.1(vi) imply that $y=S_{\alpha} u$ if $\alpha>0$. In the case of $\alpha=0$ this is obtained by Proposition 3.8. Moreover, $u \in \mathcal{C}$ since $\mathcal{C}$ is weakly closed. Finally, the weakly lower semicontinuity of $J: \mathcal{W}_{1} \times \mathcal{H} \rightarrow \mathbb{R}$ implies that $(y, u)$ is in fact a minimizer of $\left(\mathrm{P}_{\alpha}\right)$.

Next, it will be shown that a sequence $\left(y_{n}, u_{n}\right)$ of minimizers to problem $\left(\mathrm{P}_{\alpha_{n}}\right)$ for $\alpha_{n} \rightarrow 0$ admits a cluster point in a suitable topology which is a minimizer of $(\mathrm{P})$. For this purpose we have to pass to the limit in $J\left(y_{n}, u_{n}\right)$ which, in particular, requires strong convergence of $y(T)$ in $H$. This is proved next.

Lemma 3.10. Let $\left(\alpha_{n}\right)$ be a sequence in $\Lambda$ with $\alpha_{n} \rightarrow 0$, and let $\left(y_{n}, u_{n}\right) \subset \mathcal{W}_{1} \times \mathcal{H}$ be a sequence of solutions to problem $\left(\mathrm{Q}_{\alpha_{n}}\right)$ such that $\left(u_{n}\right)$ is bounded in $\mathcal{H}$. Then there exist $a \in \mathcal{H}, y \in \mathcal{W}_{1}$, and a subsequence (denoted by the index $m$ ) such that

$$
\begin{aligned}
\left(y_{m}, \widehat{\mathcal{A}}_{\alpha_{m}} y_{m}, u_{m}\right) & \rightarrow(y, \xi, u) & & \text { in } \mathcal{W}_{1} \times \mathcal{H} \times \mathcal{H}, \\
\left(y_{m}, y_{m}(T)\right) & \rightarrow(y, y(T)) & & \text { in } \mathcal{V}_{1} \times H .
\end{aligned}
$$


Proof. Since $\left(u_{n}\right)$ is bounded in $\mathcal{H}$ and due to Corollary 3.7, we can pass to a subsequence such that $u_{m} \rightarrow u$ in $\mathcal{H}$ and $\widehat{\mathcal{A}}_{\alpha_{n}} y_{m} \rightarrow \xi$ in $\mathcal{H}$ for some $u, \xi \in \mathcal{H}$. Then, Proposition 3.8 shows that $y_{m} \rightarrow y:=S u$ weakly in $\mathcal{W}_{1}$ and strongly in $\mathcal{V}_{1}$. It remains to show that $y_{m}(T)$ converges strongly to $y(T)$ in $H$. For this purpose, notice that $\mathcal{W}_{1}$ embeds continuously into $C(\overline{\mathcal{T}} ; H)$, and hence $y_{m}(T) \rightarrow y(T)$ in $H$. In order to prove the strong convergence, we show that $\left(y_{n}\right)$ is bounded in $\mathcal{V}_{2}$, and we apply a compactness argument.

As in the proof of Theorem 2.10, which is given in section $\mathrm{A}$, let $\left(e_{n}\right)$ be a complete orthonormal system of eigenvectors for the eigenvalues $\left(\lambda_{n}\right)_{n \in \mathbb{N}}$ in $V_{1}$ of the operator $L_{1}^{-1} I_{1}$ and the span of $V_{1, n}:=\operatorname{span}\left\{e_{1}, \ldots, e_{n}\right\}$. Defining $\widetilde{y}_{n, m}:=P_{V_{1, m}} y_{n}$ and $\widetilde{u}_{n, m}:=P_{V_{1, m}} u_{n}$, where $P_{V_{1, n}}$ denotes the orthogonal projection of $V_{1}$ onto $V_{1, n}$, it follows that $\widetilde{y}_{n, m} \rightarrow y_{n}$ in $\mathcal{V}_{1}$ as $m \rightarrow \infty$. Since $\left(y_{n}\right)$ solves $\left(Q_{\alpha_{n}}\right)$ for the right-hand side $u_{n} \in \mathcal{H}$ with $\widetilde{w}_{n, m}:=\mathcal{L}_{1}^{-1}\left(\widetilde{u}_{n, m}-\widetilde{y}_{n, m}^{\prime}\right)$, we obtain that

$$
\begin{aligned}
&\left(\widetilde{u}_{n, m} \mid \widetilde{y}_{n, m}\right)_{\mathcal{H}}-\frac{1}{2}\left(\left\|\widetilde{y}_{n, m}(T)\right\|_{H}^{2}-\left\|\widetilde{y}_{n, m}(0)\right\|_{H}^{2}\right) \\
& \quad=-\left(\widetilde{y}_{n, m}^{\prime}-\widetilde{u}_{n, m} \mid \widetilde{y}_{n, m}\right)_{\mathcal{H}}=\left\langle\mathcal{L}_{1} \widetilde{w}_{n, m}, \widetilde{y}_{n, m}\right\rangle_{\mathcal{V}_{1}}=\left\langle\widetilde{w}_{n, m}, \mathcal{L}_{3} \widetilde{y}_{n, m}\right\rangle_{\mathcal{V}_{1}} \\
& \quad=\left\|\mathcal{L}_{3} \widetilde{y}_{n, m}\right\|_{\mathcal{H}}^{2}+\left\langle\mathcal{A}_{\alpha_{n}} \widetilde{y}_{n, m}, \mathcal{L}_{3} \widetilde{y}_{n, m}\right\rangle_{\mathcal{V}_{1}}+\left\langle\eta \widetilde{y}_{n, m}, \mathcal{L}_{3} \widetilde{y}_{n, m}\right\rangle_{\mathcal{V}_{1}} .
\end{aligned}
$$

Because $\left(\eta \widetilde{y}_{n, m}\right)$ is bounded in $\mathcal{H}$ and $\left\langle\mathcal{A}_{\alpha_{n}} \widetilde{y}_{n, m}, \mathcal{L}_{3} \widetilde{y}_{n, m}\right\rangle_{\mathcal{V}_{1}}=\left\langle\mathcal{L}_{1} \widetilde{y}_{n, m}, \widehat{\mathcal{A}}_{\alpha_{n}} \widetilde{y}_{n, m}\right\rangle_{\mathcal{V}_{1}} \geq$ 0 , it follows that

$-C\left\|\mathcal{L}_{3} \widetilde{y}_{n, m}\right\|_{\mathcal{H}}+\left\|\mathcal{L}_{3} \widetilde{y}_{n, m}\right\|_{\mathcal{H}}^{2}+\frac{1}{2}\left\|\widetilde{y}_{n, m}(T)\right\|_{H}^{2} \leq\left\|\widetilde{u}_{n, m}\right\|_{\mathcal{H}}\left\|\widetilde{y}_{n, m}\right\|_{\mathcal{H}}+\frac{1}{2}\left\|\widetilde{y}_{n, m}(0)\right\|_{\mathcal{H}}^{2}$.

Since the right-hand side is bounded, we conclude the boundedness of $\left(\mathcal{L}_{3} \widetilde{y}_{n, m}\right)$ in $\mathcal{H}$ and therefore of $\left(\widetilde{y}_{n, m}\right)$ in $\mathcal{V}_{2}$. Thus, also $\left(y_{n}\right)$ remains bounded in $\mathcal{V}_{2}$. Applying interpolation arguments, it can be shown that $L^{2}\left(\mathcal{T} ; V_{2}\right) \cap H^{1}\left(\mathcal{T} ; V_{1}^{*}\right)$ continuously embeds into $C\left(\overline{\mathcal{T}} ; V_{1 / 2}\right)$ for a Hilbert space $V_{1 / 2}$ that is compactly embedded in $H$ (cf., e.g., [6]). This means that $y \mapsto y(T)$ is a compact mapping from $L^{2}\left(\mathcal{T} ; V_{2}\right) \cap H^{1}\left(\mathcal{T} ; V_{1}^{*}\right)$ into $H$. Consequently, after passing to a subsequence $\left(y_{l}\right)$ of $\left(y_{m}\right)$ we have that $y_{l}(T)$ converges strongly in $H$ to $y(T)$. This completes the proof.

Proposition 3.11. Let $\left(\alpha_{n}\right)$ be a sequence in $\Lambda$ with $\alpha_{n} \rightarrow 0$, and let $\left(y_{n}, u_{n}\right) \subset$ $\mathcal{W}_{1} \times \mathcal{C}$ be a sequence of minimizers to $\left(\mathrm{P}_{\alpha_{n}}\right)$. Then there exist subsequences (denoted by the index $m)$ and $(y, u) \in \mathcal{W}_{1} \times \mathcal{C}$, which is a minimizer of $(\mathrm{P})$, such that

$$
\begin{array}{clrl}
\left(y_{m}, \widehat{\mathcal{A}}_{\alpha_{m}} y_{m}\right) & \rightarrow(y, \xi) & & \text { in } \mathcal{W}_{1} \times \mathcal{H}, \\
\left(y_{m}, y_{m}(T), u_{m}\right) \rightarrow(y, y(T), u) & & \text { in } \mathcal{V}_{1} \times H \times \mathcal{H}
\end{array}
$$

Proof. For fixed $v \in \mathcal{H}$ the sequence $S_{\alpha_{n}} v$ converges weakly in $\mathcal{W}_{1}$ to $S v$ by Proposition 3.8. In particular, $\left(S_{\alpha_{n}} v\right)$ is bounded in $\mathcal{W}_{1}$, and hence it holds that

$$
J\left(y_{n}, u_{n}\right) \leq J\left(S_{\alpha_{n}} v, v\right) \leq C
$$

for some constant $C$, because $\left(y_{n}, u_{n}\right)$ is a minimizer of $\left(\mathrm{P}_{\alpha_{n}}\right)$. The coercivity of $J$ implies that $\left(u_{n}\right)$ is bounded in $\mathcal{H}$. Applying Lemma 3.10 guarantees the existence of sequences with index $m$ such that $\left(y_{m}, \widehat{\mathcal{A}}_{\alpha_{m}} y_{m}, u_{m}\right) \rightarrow(y, \xi, u)$ in $\mathcal{W}_{1} \times \mathcal{H} \times \mathcal{H}$ and $\left(y_{m}, y_{m}(T)\right) \rightarrow(y, y(T))$ in $\mathcal{V}_{1} \times H$. Note that $(y, u)$ satisfies $u \in \mathcal{C}$ and $S u=y$ by Proposition 3.8 .

Now, let $\left(y^{*}, u^{*}\right) \in \mathcal{W}_{1} \times \mathcal{C}$ be a minimizer of $(\mathrm{P})$ (which exists by virtue of Proposition 3.9). From Proposition 3.8 and Theorem 3.3 we conclude that $\left(S_{\alpha_{m}} u^{*}, u^{*}\right) \rightarrow$ 
$\left(y^{*}, u^{*}\right)$ in $\mathcal{W}_{1} \times \mathcal{H}$ and $S_{\alpha_{m}} u^{*} \rightarrow y^{*}$ in $\mathcal{V}_{1}$ for a suitable subsequence. By Lemma 3.10 and after passing to another subsequence, which we still denote by the index $m$, we have that $S_{\alpha_{m}} u^{*}(T) \rightarrow y^{*}(T)$ in $H$. The weak lower semicontinuity of $J$ and the convergence properties of $\left(S_{\alpha_{m}} u^{*}, u^{*}\right)$ imply that

$$
\begin{aligned}
J\left(y^{*}, u^{*}\right) & \leq J(y, u) \leq \varliminf_{m \rightarrow \infty} J\left(y_{m}, u_{m}\right) \leq \varlimsup_{m \rightarrow \infty} J\left(y_{m}, u_{m}\right) \leq \varlimsup_{m \rightarrow \infty} J\left(S_{\alpha_{m}} u^{*}, u^{*}\right) \\
& =J\left(y^{*}, u^{*}\right) .
\end{aligned}
$$

Consequently, $(y, u)$ is a minimizer of $(\mathrm{P})$. Moreover, we have that $J\left(y_{m}, u_{m}\right) \rightarrow J(y, u)$ which implies $\left\|u_{m}\right\|_{\mathcal{H}} \rightarrow\|u\|_{\mathcal{H}}$. Since we already know that $u_{m} \rightarrow u$ in $\mathcal{H}$, it follows that $u_{m} \rightarrow u$ in $\mathcal{H}$.

\subsection{Stationarity system for $\left(\mathbf{P}_{\alpha}\right)$.}

TheOREM 3.12. For every $\alpha \in \Lambda$ and for every minimizer $\left(y_{\alpha}, u_{\alpha}\right) \in \mathcal{W}_{1} \times \mathcal{H}$ of problem $\left(\mathrm{P}_{\alpha}\right)$, there exists a $p_{\alpha} \in \mathcal{W}_{3}$ with

$$
\begin{gathered}
-p_{\alpha}^{\prime}+\mathcal{L}_{1} \mathcal{L}_{3} p_{\alpha}+\mathcal{R}_{\alpha}^{*} \mathcal{L}_{3} p_{\alpha}=\mu_{1}\left(y_{\alpha}-y_{\Omega}\right), \\
p_{\alpha}(T)=\mu_{2}\left(y_{\alpha}(T)-y_{T}\right),
\end{gathered}
$$

where $\mathcal{R}_{\alpha}:=\overline{D \mathcal{A}_{\alpha}}\left(y_{\alpha}\right)+\eta \mathcal{I} \in \mathcal{L}\left(\mathcal{V}_{1} ; \mathcal{V}_{1}\right)$. Moreover, for $\mathcal{C}_{\alpha}:=\mathbb{R}^{+}\left(\mathcal{C}-u_{\alpha}\right)$ we have

$$
p_{\alpha}+u_{\alpha} \in \mathcal{C}_{\alpha}^{+}
$$

Proof. First, recall that by Remark 3.4 a minimizer $\left(y_{\alpha}, u_{\alpha}\right) \in \mathcal{W}_{1} \times \mathcal{H}$ satisfies $y_{\alpha} \in \mathcal{W}_{4}$, and therefore $\mathcal{R}_{\alpha}$ is indeed a linear, bounded operator on $\mathcal{V}_{1}$ by Assumption 3.1(iv).

In order to prove the assertion, we first apply a theorem of Zowe and Kurcyusz [63] which guarantees the existence of a Lagrange multiplier $p_{\alpha}$ satisfying a particular partial differential equation. In the second step we show the relation between this multiplier and the control $u_{\alpha}$, and in the last step we prove that $p_{\alpha}$ indeed is a solution to the evolution equation above.

1. For $v_{0} \in V_{1}$ we define $\mathcal{W}_{3}\left(v_{0}\right):=\left\{v \in \mathcal{W}_{3}: v(0)=v_{0}\right\}$ and consider the following setting in order to apply Theorem 3.1 of [63]:

$$
\begin{aligned}
X & :=\mathcal{W}_{3} \times \mathcal{H}, & C_{X} & :=\mathcal{W}_{3}\left(y_{0}\right) \times \mathcal{C}, \\
Y & :=\mathcal{V}_{1}^{*}, & K & :=\{0\} \subset Y, \\
g & : X \rightarrow Y, & g(y, u) & :=y^{\prime}+\mathcal{L}_{1}\left(\mathcal{L}_{3}+\widehat{\mathcal{A}}_{\alpha}+\eta \mathcal{I}\right) y-u, \\
f & : X \rightarrow \mathbb{R}, & f(y, u) & :=J(y, u) .
\end{aligned}
$$

From Assumption 3.1 it follows that $f$ and $g$ are continuously Fréchet differentiable with

$$
\begin{gathered}
D g\left(y, u ; \delta_{y}, \delta_{u}\right)=\delta_{y}{ }^{\prime}+\mathcal{L}_{1}\left(\mathcal{L}_{3}+D \widehat{\mathcal{A}}_{\alpha}(y)+\eta \mathcal{I}\right) \delta_{y}-\delta_{u} \\
D f\left(y, u ; \delta_{y}, \delta_{u}\right)=\mu_{1}\left(y-y_{\Omega} \mid \delta_{y}\right)_{\mathcal{H}}+\mu_{2}\left(y(T)-y_{T} \mid \delta_{y}(T)\right)_{H}+\left(u \mid \delta_{u}\right)_{\mathcal{H}}
\end{gathered}
$$

for $\left(\delta_{y}, \delta_{u}\right) \in \mathcal{W}_{3} \times \mathcal{H}$. For given $v^{*} \in Y$ we choose $u:=0 \in \mathcal{H}$ and $y \in \mathcal{W}_{1}$ to be the solution of

$$
y^{\prime}+\mathcal{L}_{1} w=v^{*}, \quad w=\left(\mathcal{L}_{1}+\mathcal{R}_{\alpha}\right) y, \quad y(0)=0
$$


Due to Theorem 2.7 such a solution exists since $\mathcal{R}_{\alpha}: \mathcal{V}_{1} \rightarrow \mathcal{V}_{1}^{*}$ is Lipschitz continuous. Moreover, Theorem 2.10 shows that $y \in \mathcal{W}_{3}$ as well as

$$
y^{\prime}+\mathcal{L}_{1}\left(\mathcal{L}_{3}+\mathcal{R}_{\alpha}\right) y-u=v^{*} .
$$

This demonstrates that $g^{\prime}(\widetilde{x}) \in \mathcal{L}\left(\mathcal{W}_{3}(0) \times\{0\} ; Y\right)$ is surjective for $\widetilde{x}:=\left(y_{\alpha}, u_{\alpha}\right)$. Now, using Theorem 3.1 of Zowe and Kurcyusz [63], there is a $\widehat{p_{\alpha}} \in Y^{*}$ such that

$$
\left\langle\widehat{p_{\alpha}}, g(\widetilde{x})\right\rangle_{Y}=0, \quad \operatorname{Df}\left(\widetilde{x} ; \delta_{y}, \delta_{u}\right)-\left\langle\widehat{p_{\alpha}}, D g\left(\widetilde{x} ; \delta_{y}, \delta_{u}\right)\right\rangle_{Y}=0
$$

for all $\left(\delta_{y}, \delta_{u}\right) \in X_{0}:=\mathcal{W}_{3}(0) \times \mathcal{C}_{\alpha}$. Since $Y^{*} \cong \mathcal{V}_{1}$, the multiplier $\widehat{p_{\alpha}} \in Y^{*}$ can be identified with a $p_{\alpha} \in \mathcal{V}_{1}$ satisfying

$$
\begin{gathered}
\left\langle y_{\alpha}{ }^{\prime}+\mathcal{L}_{1}\left(\mathcal{L}_{3}+\widehat{\mathcal{A}}_{\alpha}+\eta \mathcal{I}\right) y_{\alpha}-u_{\alpha}, p_{\alpha}\right\rangle_{\mathcal{V}_{1}}=0, \\
D J\left(\widetilde{x} ; \delta_{y}, \delta_{u}\right)-\left\langle\delta_{y}{ }^{\prime}+\mathcal{L}_{1}\left(\mathcal{L}_{3}+\mathcal{R}_{\alpha}\right) \delta_{y}-\delta_{u}, p_{\alpha}\right\rangle_{\mathcal{V}_{1}}=0
\end{gathered}
$$

for all $\left(\delta_{y}, \delta_{u}\right) \in X_{0}$.

2. By choosing $\delta_{y}=0$ in (3.2) and since $D J\left(\widetilde{x} ; 0, \delta_{u}\right)=\left(u_{\alpha} \mid \delta_{u}\right)_{\mathcal{H}}$ we find

$$
D J\left(\widetilde{x} ; 0, \delta_{u}\right)+\left\langle\delta_{u}, p_{\alpha}\right\rangle_{\mathcal{V}_{1}}=\left(u_{\alpha}+p_{\alpha} \mid \delta_{u}\right)_{\mathcal{H}}=0
$$

for all $\delta_{u} \in \mathcal{C}_{\alpha}$. This yields $p_{\alpha}+u_{\alpha} \in \mathcal{C}_{\alpha}^{+} \subset \mathcal{H}$.

3. Now we show that (3.1) and (3.2) imply the assertion on $p_{\alpha}$. For this purpose, first recall that a function $z \in L^{2}(\mathcal{T} ; Z)$ has a weak derivative $v \in L^{2}(\mathcal{T} ; Z)$ for some Banach space $Z$ if and only if

$$
\int_{\mathcal{T}} \eta v=-\int_{\mathcal{T}} \eta^{\prime} z \quad \forall \eta \in C_{c}^{\infty}(\mathcal{T})
$$

From (3.2) and the symmetry of $\mathcal{L}_{1}$ it follows that for all $\left(\delta_{y}, \delta_{u}\right) \in X_{0}$ it holds that

$$
\begin{aligned}
\left\langle\delta_{y}{ }^{\prime}, p_{\alpha}\right\rangle_{\mathcal{V}_{1}} & =\operatorname{DJ}\left(\widetilde{x} ; \delta_{y}, \delta_{u}\right)-\left\langle\mathcal{L}_{1} p_{\alpha},\left(\mathcal{L}_{3}+\mathcal{R}_{\alpha}\right) \delta_{y}\right\rangle_{\mathcal{V}_{1}}+\left\langle\delta_{u}, p_{\alpha}\right\rangle_{\mathcal{V}_{1}} \\
& =\operatorname{DJ}\left(\widetilde{x} ; \delta_{y}, \delta_{u}\right)-\left\langle\left(\mathcal{L}_{3}+\mathcal{R}_{\alpha}\right)^{*} \mathcal{L}_{1} p_{\alpha}, \delta_{y}\right\rangle_{\mathcal{V}_{3}}+\left\langle\delta_{u}, p_{\alpha}\right\rangle_{\mathcal{V}_{1}} .
\end{aligned}
$$

Now, for arbitrary $v_{0} \in V_{3}$ and $\eta \in C_{c}^{\infty}(\mathcal{T})$, by choosing $\delta_{y}(t):=\eta(t) v_{0}, \delta_{u}:=0$ and defining $q:=\mu_{1}\left(y_{\alpha}-y_{\Omega}\right)-\left(\mathcal{L}_{3}+\mathcal{R}_{\alpha}\right)^{*} \mathcal{L}_{1} p_{\alpha} \in \mathcal{V}_{3}^{*}$ we obtain

$$
\left\langle\eta^{\prime} v_{0}, p_{\alpha}\right\rangle_{\mathcal{V}_{1}}=\left\langle q, \eta v_{0}\right\rangle_{\mathcal{V}_{3}}
$$

and therefore

$$
\left\langle\int_{\mathcal{T}} \eta^{\prime} p_{\alpha}-\int_{\mathcal{T}} \eta q, v_{0}\right\rangle_{V_{3}}=\left\langle\eta^{\prime} v_{0}, p_{\alpha}\right\rangle_{\mathcal{V}_{1}}-\left\langle q, \eta v_{0}\right\rangle_{\mathcal{V}_{3}}=0
$$

Since $v_{0} \in V_{3}$ was arbitrary, $\int_{\mathcal{T}} \eta^{\prime} p_{\alpha}=\int_{\mathcal{T}} \eta q$ holds for every $\eta \in C_{c}^{\infty}(\mathcal{T})$. This implies that $p_{\alpha} \in H^{1}\left(\mathcal{T} ; V_{3}^{*}\right)$ and

$$
p_{\alpha}^{\prime}=\left(\mathcal{L}_{3}+\mathcal{R}_{\alpha}\right)^{*} \mathcal{L}_{1} p_{\alpha}-\mu_{1}\left(y_{\alpha}-y_{\Omega}\right) .
$$

Therefore, in the case $\delta_{u}=0,(3.2)$ reduces to

$$
\left\langle\delta_{y}{ }^{\prime}, p_{\alpha}\right\rangle_{\mathcal{V}_{1}}+\left\langle p_{\alpha}{ }^{\prime}, \delta_{y}\right\rangle_{\mathcal{V}_{3}}-\mu_{2}\left(y_{\alpha}(T)-y_{T} \mid \delta_{y}(T)\right)_{H}=0
$$


With the help of Proposition 2.9 we infer that

$$
\left\langle p_{\alpha}(T), \delta_{y}(T)\right\rangle_{V_{1}}-\left\langle p_{\alpha}(0), \delta_{y}(0)\right\rangle_{V_{1}}-\mu_{2}\left\langle y_{\alpha}(T)-y_{T}, \delta_{y}(T)\right\rangle_{V_{1}}=0
$$

for all $\delta_{y} \in \mathcal{W}_{3}(0)$. Since it is possible to find a sequence $\delta_{y_{n}} \in \mathcal{W}_{3}(0)$ such that $\delta_{y_{n}}(T) \rightarrow v_{1}$ in $V_{1}$ for arbitrarily given $v_{1} \in V_{1}$, we conclude that

$$
p_{\alpha}(T)=\mu_{2}\left(y_{\alpha}(T)-y_{T}\right) .
$$

Finally, applying Theorem 2.10 again finishes the proof.

Lemma 3.13. Let $y \in \mathcal{W}_{3}, \alpha \in \Lambda$. Then $\mathcal{I}_{1} \overline{D \mathcal{A}_{\alpha}}(y): \mathcal{V}_{1} \rightarrow \mathcal{V}_{1}^{*}$ is monotone.

Proof. Let $v \in \mathcal{V}_{1}$ be given. We have to show that $\left\langle\overline{D \mathcal{A}_{\alpha}}(y) v, v\right\rangle_{\mathcal{V}_{1}} \geq 0$. Since the image of $I_{\mathcal{W}_{3} \rightarrow \mathcal{V}_{1}}$ is dense in $\mathcal{V}_{1}$, we may find a sequence $\left(y_{n}\right)$ in $\mathcal{W}_{3}$ with $y_{n} \rightarrow v$ in $\mathcal{V}_{1}$. By the continuity of $\overline{D \mathcal{A}_{\alpha}}(y)$ and the Fréchet differentiability of $\widehat{\mathcal{A}}_{\alpha}: \mathcal{W}_{3} \rightarrow \mathcal{V}_{1}$ in $y$, it holds that

$$
\begin{aligned}
\left\langle\overline{D \mathcal{A}_{\alpha}}(y) v, v\right\rangle_{\mathcal{V}_{1}} & =\lim _{n \rightarrow \infty}\left\langle D \widehat{\mathcal{A}}_{\alpha}(y) y_{n}, y_{n}\right\rangle_{\mathcal{V}_{1}} \\
& =\lim _{n \rightarrow \infty} \lim _{t \rightarrow 0} \frac{1}{t}\left\langle\widehat{\mathcal{A}}_{\alpha}\left(y+t y_{n}\right)-\widehat{\mathcal{A}}_{\alpha} y, y_{n}\right\rangle_{\mathcal{V}_{1}} \\
& =\lim _{n \rightarrow \infty} \lim _{t \rightarrow 0} \frac{1}{t^{2}}\left\langle\mathcal{A}_{\alpha}\left(y+t y_{n}\right)-\mathcal{A}_{\alpha} y,\left(y+t y_{n}\right)-y\right\rangle_{\mathcal{V}_{1}} \\
& \geq 0
\end{aligned}
$$

since $A_{\alpha}=\partial \varphi_{\alpha}$, which is monotone (cf. [10, Proposition 2.1]).

3.3. The limit problem. We conclude this section by establishing an optimality system for the limit problem. It involves the notion of derivatives of vector-valued distributions which we recall first (cf. [29] and [12] for further details).

Definition 3.14. Let $\mathcal{D}(\mathcal{T}):=C_{c}^{\infty}(\mathcal{T})$ denote the space of test functions with the usual locally convex topology. For a Banach space $X$, the space of $X$-valued distributions on $\mathcal{T}$ is the space of all linear, continuous mappings $\mathcal{D}^{*}(\mathcal{T} ; X):=\mathcal{L}\left(\mathcal{D}(\mathcal{T}) ; X_{w}\right)$ from $\mathcal{D}(\mathcal{T})$ into $X$ equipped with its weak topology $X_{w}$. The topology of $\mathcal{D}^{*}(\mathcal{T} ; X)$ is chosen to be the topology induced by the system $\left\{p_{w, x^{*}} \mid w \in \mathcal{D}(\mathcal{T}), x^{*} \in X^{*}\right\}$ of seminorms given for $v \in \mathcal{D}^{*}(\mathcal{T} ; X)$ by

$$
p_{w, x^{*}}(v):=\left|\left\langle x^{*}, v(w)\right\rangle_{X}\right| .
$$

Moreover, the derivative' $: \mathcal{D}^{*}(\mathcal{T} ; X) \rightarrow \mathcal{D}^{*}(\mathcal{T} ; X)$ of $X$ is defined for $w \in \mathcal{D}(\mathcal{T})$ by

$$
v^{\prime}(w):=-v\left(w^{\prime}\right)
$$

Remark 3.15. As usual, a locally integrable function $f \in L_{\text {loc }}^{1}(\mathcal{T} ; X)$ can be identified with the distribution $F_{f} \in \mathcal{D}^{*}(\mathcal{T} ; X)$ given by

$$
F_{f}(w):=\int_{\Omega} w f \quad \text { for } w \in \mathcal{D}(\mathcal{T})
$$

THEOREM 3.16. Let $\left(\alpha_{n}\right)$ be a sequence in $\Lambda$ with $\alpha_{n} \rightarrow 0$. We assume that $\left(y_{n}, u_{n}\right) \in \mathcal{W}_{3} \times \mathcal{C}$ is a sequence of minimizers to problem $\left(\mathrm{P}_{\alpha_{n}}\right)$, that $p_{n} \in \mathcal{W}_{3}$ and $\mathcal{C}_{\alpha} \subset \mathcal{H}$ are given as in Theorem $3.12, w_{n}:=\mathcal{L}_{1}^{-1}\left(u_{n}-y_{n}^{\prime}\right), \quad \xi_{n}:=\widehat{\mathcal{A}}_{\alpha_{n}} y_{n} \in \mathcal{V}_{1}, \quad \lambda_{n}:=$ 
$\overline{D \mathcal{A}_{\alpha_{n}}}\left(y_{n}\right)^{*} \mathcal{L}_{3} p_{n} \in \mathcal{V}_{1}^{*}$, and $\kappa_{n}:=\eta \mathcal{I}_{1} \mathcal{L}_{3} p_{n} \in \mathcal{V}_{1}^{*}$. Then there exist subsequences (denoted by the index $m$ ) and a minimizer $(y, u) \in \mathcal{W}_{1} \times \mathcal{C}$ of $(\mathrm{P})$ such that

$$
\begin{aligned}
& \left(y_{m}, w_{m}, p_{m}, p_{m}^{\prime}, p_{m}(0)\right) \rightarrow\left(y, w, p, p^{\prime}, p_{0}\right) \quad \text { in } \mathcal{W}_{1} \times \mathcal{V}_{1} \times \mathcal{V}_{3} \times \mathcal{W}_{1}^{*} \times V_{1}, \\
& \left(\lambda_{m}, \xi_{m}, \kappa_{m}\right) \rightarrow(\lambda, \xi, \kappa) \quad \text { in } \mathcal{W}_{1}^{*} \times \mathcal{H} \times \mathcal{V}_{1}^{*} \text {, } \\
& \left(y_{m}, u_{m}\right) \rightarrow(y, u) \quad \text { in } \mathcal{V}_{1} \times \mathcal{H} \text {, } \\
& p_{m} \stackrel{*}{\rightarrow} p \quad \text { in } L^{\infty}\left(\mathcal{T} ; V_{1}\right),
\end{aligned}
$$

where $p^{\prime}$ denotes the distributional derivative of $p \in \mathcal{D}^{*}\left(\mathcal{T} ; V_{1}\right)$. It holds that $y_{n}(0)=$ $y_{0}, p_{n}(T)=\mu_{2}\left(y_{n}(T)-y_{T}\right), p_{n}+u_{n} \in \mathcal{C}_{\alpha}^{+}$,

$$
\begin{gathered}
y_{n}^{\prime}+\mathcal{L}_{1} w_{n}=u_{n}, \quad w_{n}=\mathcal{L}_{3} y_{n}+\xi_{n}+\eta \mathcal{I} y_{n}, \\
-p_{n}^{\prime}+\mathcal{L}_{1} \mathcal{L}_{3} p_{n}+\lambda_{n}+\kappa_{n}=\mu_{1}\left(y_{n}-y_{\Omega}\right),
\end{gathered}
$$

as well as $y(0)=y_{0}, p(T)=\mu_{2}\left(y(T)-y_{T}\right), p+u \in \mathcal{C}_{0}^{+}$for $\mathcal{C}_{0}:=\mathbb{R}^{+}(\mathcal{C}-u)$, and

$$
\begin{gathered}
y^{\prime}+\mathcal{L}_{1} w=u, \quad w=\mathcal{L}_{1} y+\xi+\eta \mathcal{I}_{1} y, \\
-p^{\prime}+\mathcal{L}_{1} \mathcal{L}_{3} p+\lambda+\kappa=\mu_{1}\left(y-y_{\Omega}\right) .
\end{gathered}
$$

Proof. Since $\left(y_{n}\right)$ and $y$ are solutions to $\left(\mathrm{P}_{\alpha_{n}}\right)$ and $(\mathrm{P})$, respectively, (3.3) and (3.5) are satisfied together with the corresponding initial conditions. Theorem 3.12 guarantees (3.4) and $p_{n}(T)=\mu_{2}\left(y_{n}(T)-y_{T}\right)$. The boundedness and convergence properties of $\left(y_{m}\right),\left(w_{m}\right),\left(\xi_{m}\right)$, and $\left(u_{m}\right)$ are obtained from Corollary 3.7 and Proposition 3.11. Let $\eta_{-}:=\max (1,-\eta)$. Then

$$
\left\langle\eta \mathcal{I}_{1} v, v\right\rangle_{\mathcal{V}_{1}} \geq-\eta_{-}\|v\|_{\mathcal{H}}^{2}
$$

holds for all $v \in \mathcal{V}_{1}$. For the time being, let us fix $t_{0} \in \overline{\mathcal{T}}$ and define $\varphi: \overline{\mathcal{T}} \rightarrow$ $\mathbb{R}, \varphi(t):=\exp \left(\eta_{-}^{2} t\right)$ together with $\widetilde{p_{n}}:=\varphi p_{n} \in \mathcal{W}_{3}, \widetilde{q_{n}}:=\chi_{\left[t_{0}, T\right]} \widetilde{p_{n}} \in \mathcal{V}_{3}$, and $f_{n}:=\varphi\left(y_{n}-y_{\Omega}\right) \in \mathcal{H}$. Using (3.4) we obtain that

$$
\begin{aligned}
\widetilde{p_{n}} & =\varphi\left(\eta_{-}^{2} p_{n}+p_{n}^{\prime}\right) \\
& =\varphi\left(\eta_{-}^{2} p_{n}+\left[\mathcal{L}_{1}+\overline{D \mathcal{A}_{\alpha_{n}}}\left(y_{n}\right)^{*}+\eta \mathcal{I}_{1}\right] \mathcal{L}_{3} p_{n}\right)-f_{n} \\
& =\eta_{-}^{2} \widetilde{p_{n}}+\left[\mathcal{L}_{1}+\overline{D \mathcal{A}_{\alpha_{n}}}\left(y_{n}\right)^{*}+\eta \mathcal{I}_{1}\right] \mathcal{L}_{3} \widetilde{p_{n}}-f_{n}
\end{aligned}
$$

The integration-by-parts formula yields

$$
\left\langle\widetilde{p_{n}}, \mathcal{L}_{3} \widetilde{q_{n}}\right\rangle_{\mathcal{V}_{1}}=\left\langle\left(\mathcal{I}_{3}^{*} \mathcal{L}_{1} \mathcal{I}_{3} \widetilde{p_{n}}\right)^{\prime}, \widetilde{q_{n}}\right\rangle_{\mathcal{V}_{3}}=\frac{1}{2}\left(\left\|\widetilde{p_{n}}(T)\right\|_{V_{1}}^{2}-\left\|\widetilde{p_{n}}\left(t_{0}\right)\right\|_{V_{1}}^{2}\right),
$$

and therefore, after testing $\widetilde{p_{n}}{ }^{\prime}$ with $\mathcal{L}_{3} \widetilde{q_{n}}$, we obtain with the help of Lemma 3.13 that

$$
\begin{aligned}
& \frac{1}{2}\left(\left\|\widetilde{p_{n}}(T)\right\|_{V_{1}}^{2}-\left\|\widetilde{p_{n}}\left(t_{0}\right)\right\|_{V_{1}}^{2}\right) \\
& =\left\langle{\widetilde{p_{n}}}^{\prime}, \mathcal{L}_{3} \widetilde{q_{n}}\right\rangle_{\mathcal{V}_{1}} \\
& \geq \eta_{-}^{2}\left\langle\widetilde{p_{n}}, \mathcal{L}_{3} \widetilde{q_{n}}\right\rangle_{\mathcal{V}_{1}}+\left\langle\mathcal{L}_{1} \mathcal{L}_{3} \widetilde{p_{n}}, \mathcal{L}_{3} \widetilde{q_{n}}\right\rangle_{\mathcal{V}_{1}}-\eta_{-}|| \mathcal{L}_{3} \widetilde{q_{n}}\left\|_{\mathcal{H}}^{2}-\right\| f_{n}\left\|_{\mathcal{V}_{1}^{*}}\right\| \mathcal{L}_{3} \widetilde{q_{n}} \|_{\mathcal{V}_{1}}
\end{aligned}
$$

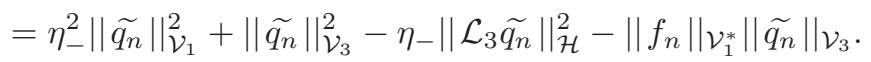


With the estimation

$$
\begin{aligned}
\left\|\mathcal{L}_{3} \widetilde{q_{n}}\right\|_{\mathcal{H}}^{2} & =\left\langle\mathcal{I}_{1} \mathcal{L}_{3} \widetilde{q_{n}}, \mathcal{L}_{3} \widetilde{q_{n}}\right\rangle_{\mathcal{V}_{1}}=\left\langle\mathcal{L}_{3}^{*} \mathcal{I}_{1} \mathcal{L}_{3} \widetilde{q_{n}}, \widetilde{q_{n}}\right\rangle_{\mathcal{V}_{1}}=\left\langle\mathcal{L}_{1} \mathcal{L}_{3} \widetilde{q_{n}}, \mathcal{I}_{3} \widetilde{q_{n}}\right\rangle_{\mathcal{V}_{1}} \\
& \leq\left\|\mathcal{L}_{1} \mathcal{L}_{3} \widetilde{q_{n}}\right\|_{\mathcal{V}_{1}^{*}}\left\|\widetilde{q_{n}}\right\|_{\mathcal{V}_{1}} \leq \frac{1}{2 \eta_{-}}\left\|\widetilde{q_{n}}\right\|_{\mathcal{V}_{3}}^{2}+\frac{\eta_{-}}{2}\left\|\widetilde{q_{n}}\right\|_{\mathcal{V}_{1}}^{2}
\end{aligned}
$$

we finally arrive at

$$
\left\|\widetilde{p_{n}}(T)\right\|_{V_{1}}^{2} \geq\left\|\widetilde{p_{n}}\left(t_{0}\right)\right\|_{V_{1}}^{2}+\eta_{-}^{2}\left\|\widetilde{q_{n}}\right\|_{\mathcal{V}_{1}}^{2}+\left\|\widetilde{q_{n}}\right\|_{\mathcal{V}_{3}}^{2}-2\left\|f_{n}\right\|\left\|_{\mathcal{V}_{1}^{*}}\right\| \widetilde{q_{n}} \|_{\mathcal{V}_{3}} .
$$

By Corollary 3.7, $y_{n}$ is bounded in $L^{\infty}\left(\mathcal{T} ; V_{1}\right)$. Hence $p_{n}(T)=\mu_{2}\left(y_{n}(T)-y_{T}\right)$ in $V_{1}$. Since $\left(f_{n}\right)$ is bounded in $\mathcal{V}_{1}^{*}$, it follows that $\left(\widetilde{p_{n}}\right)$ and thus also $\left(p_{n}\right)$ are bounded in $\mathcal{V}_{3}$ (by choosing $t_{0}=0$ ) as well as $\left(p_{n}\left(t_{0}\right)\right)$ in $V_{1}$. For $v \in \mathcal{W}_{1}$ we have that

$$
\begin{aligned}
\left\langle p_{n}^{\prime}, v\right\rangle_{\mathcal{W}_{1}} & =\left\langle p_{n}^{\prime}, v\right\rangle_{\mathcal{V}_{1}}=-\left\langle v^{\prime}, p_{n}\right\rangle_{\mathcal{V}_{1}}+\left(p_{n}(T) \mid v(T)\right)_{H}-\left(p_{n}(0) \mid v(0)\right)_{H} \\
& \leq C\left(\left\|p_{n}\right\|_{\mathcal{V}_{1}}+\left\|p_{n}(T)\right\|_{H}+\left\|p_{n}(0)\right\|_{H}\right)\|v\|_{\mathcal{W}_{1}},
\end{aligned}
$$

which shows that $p_{n}^{\prime}$ is bounded in $\mathcal{W}_{1}^{*}$. The boundedness of $\left(p_{n}\right)$ in $\mathcal{V}_{3}$, together with Assumption 3.1, implies that $\left(\kappa_{n}\right)$ is bounded in $\mathcal{V}_{1}^{*}$. Moreover, the embedding of $\mathcal{V}_{1}^{*}$ into $\mathcal{W}_{1}^{*}$ is continuous, and hence it follows from (3.4) that also $\left(\lambda_{n}\right)$ is bounded in $\mathcal{W}_{1}^{*}$.

Hence, we can pass to a (weakly) convergent subsequence (denoted by the index $m$ ) such that all the convergences given in the assertion are fulfilled. Passing to the limit as $m \rightarrow \infty$ in (3.4) yields (3.6). To show that $p^{\prime}$ is indeed the distributional derivative of $p$, we observe that

$$
\begin{aligned}
\left\langle p^{\prime}(\varphi), v\right\rangle_{V_{1}} & =-\left\langle p\left(\varphi^{\prime}\right), v\right\rangle_{V_{1}}=-\left\langle\int_{\mathcal{T}} \varphi^{\prime} p, v\right\rangle_{V_{1}}=-\left\langle p, \varphi^{\prime} v\right\rangle_{\mathcal{V}_{1}} \\
& =-\lim \left\langle p_{m}, \varphi^{\prime} v\right\rangle_{\mathcal{V}_{1}}=\lim \left\langle p_{m}^{\prime}, \varphi v\right\rangle_{\mathcal{V}_{1}}=\lim \left\langle p_{m}^{\prime}, \varphi v\right\rangle_{\mathcal{W}_{1}} \\
& =\left\langle\lim p_{m}^{\prime}, \varphi v\right\rangle_{\mathcal{W}_{1}}
\end{aligned}
$$

for all $\varphi \in \mathcal{D}(\mathcal{T})$ and $v \in V_{1}$. This completes the proof.

4. The Cahn-Hilliard system with double-obstacle homogeneous free energy density. In this section we highlight the special case where $\varphi$ is given as the indicator function of some convex subset of $V_{1}$. This corresponds to the Cahn-Hilliard system with double-obstacle potential. Moreover, the $\varphi_{\alpha}$ are defined as mollified versions of the Moreau-Yosida approximations of $\varphi$. In this setting, a function space version of C-stationarity is obtained.

Example 4.1 (double-obstacle potential). Suppose that $N \leq 3, M=[a, b] \subset$ $\mathbb{R}, a<0<b$, is a bounded interval and

$$
\begin{aligned}
K_{H} & :=\{v \in H: v(x) \in M \text { for a.e. } x \in \Omega\}, \quad K_{V}:=I_{V_{1}}^{-1} K_{H}, \\
\mathcal{C} & \subset \mathcal{H} \text { closed, convex, and nonempty, } \\
\tilde{\gamma} & :=\imath_{M}: \mathbb{R} \rightarrow \overline{\mathbb{R}}, \quad \tilde{\beta}:=\partial \tilde{\gamma}, \\
\varphi & :=\imath_{K_{V}}: V_{1} \rightarrow \overline{\mathbb{R}}, \quad \eta:=-1, \quad \psi=\frac{1}{2}\|\cdot\|_{H}^{2}: V_{1} \rightarrow \overline{\mathbb{R}} .
\end{aligned}
$$

Let $\rho \in C^{2}(\mathbb{R})$ be a fixed mollifier with $\operatorname{supp} \rho \subset[-1,1], \int_{\mathbb{R}} \rho=1, \bar{\rho}:=\int_{\mathbb{R}} r \rho(r) d r, 0 \leq$ $\rho(r) \leq 1$ for all $r \in \mathbb{R}$. Moreover, $\varepsilon: \mathbb{R}^{+} \rightarrow \mathbb{R}^{+}$is a function with $\varepsilon(\alpha)>0, \varepsilon(\alpha) \rightarrow 0$, 
and $\frac{\varepsilon(\alpha)}{\alpha} \rightarrow 0$ as $\alpha \rightarrow 0$. By $\tilde{\gamma}_{\alpha}$ and $\tilde{\varphi_{\alpha}}$ we denote the Moreau-Yosida approximations of $\tilde{\gamma}$ and $\varphi$ with the parameter $\alpha>0$, respectively, and the Yosida approximation of the operator $\tilde{\beta}$ by $\tilde{\beta}_{\alpha}$. For the general definitions of the Moreau-Yosida and the Yosida approximation we refer the reader to [10]. We set

$$
\rho_{\varepsilon}(r):=\frac{1}{\varepsilon} \rho\left(\frac{r}{\varepsilon}\right), \quad \beta_{\alpha}:=\tilde{\beta}_{\alpha} * \rho_{\varepsilon(\alpha)}, \quad \gamma_{\alpha}(r):=\int_{0}^{r} \beta_{\alpha}, \quad \varphi_{\alpha}(u):=\int_{\Omega} \gamma_{\alpha} \circ u,
$$

where $*$ denotes the convolution operator and $f \circ g$ denotes the superposition of $f$ and $g$. Furthermore, $q: \mathbb{R} \rightarrow \mathbb{R}$ is given by $q:=\tilde{\beta}_{1}=\alpha \tilde{\beta}_{\alpha}, Q: H \rightarrow H$ denotes its superposition operator with respect to $\Omega$, and $\mathcal{Q}: \mathcal{H} \rightarrow \mathcal{H}$ denotes the superposition operator of $Q$ with respect to $\mathcal{T}$. Finally, let $y_{0} \in V_{1} \cap L^{\infty}(\Omega)$ be such that $a<$ $\operatorname{ess} \inf _{\Omega} y_{0} \leq \operatorname{ess}_{\sup _{\Omega}} y_{0}<b$.

In order to prove that Example 4.1 falls into the framework of Assumption 3.1, we first collect some basic properties.

Lemma 4.2. Given the setting of Example 4.1 we have

$$
\begin{gathered}
\tilde{\beta}_{\alpha}(r)=\left\{\begin{array}{ll}
\frac{1}{\alpha}(r-a) & \text { if } r<a, \\
0 & \text { if } a \leq r \leq b, \\
\frac{1}{\alpha}(r-b) & \text { if } b<r,
\end{array} \quad \tilde{\beta}_{\alpha}^{\prime}(r)= \begin{cases}\frac{1}{\alpha} & \text { if } r<a, \\
0 & \text { if } a<r<b, \\
\frac{1}{\alpha} & \text { if } b<r,\end{cases} \right. \\
\tilde{\gamma}_{\alpha}(r)= \begin{cases}\frac{1}{2 \alpha}(r-a)^{2} & \text { if } r<a, \\
0 & \text { if } a \leq r \leq b, \\
\frac{1}{2 \alpha}(r-b)^{2} & \text { if } b<r .\end{cases}
\end{gathered}
$$

Moreover, $\tilde{\beta}_{\alpha}$ and $\beta_{\alpha}$ are Lipschitz continuous with constant $\frac{1}{\alpha}$ and monotone, and $\beta_{\alpha}(0)=0$ if $\varepsilon(\alpha) \leq \min (-a, b)$. Furthermore, $\beta_{\alpha}(r)=\tilde{\beta}_{\alpha}(r)-\frac{\varepsilon(\alpha)}{\alpha} \bar{\rho}$ for $r \leq a-\varepsilon(\alpha)$ and $r \geq b+\varepsilon(\alpha)$, and

$$
\left|\beta_{\alpha}^{\prime}(r)\right| \leq \frac{1}{\alpha}, \quad\left|\beta_{\alpha}(r)-\tilde{\beta}_{\alpha}(r)\right| \leq \frac{\varepsilon(\alpha)}{\alpha}(2+|\bar{\rho}|), \quad\left|\beta_{\alpha}(r)-\beta_{\alpha}^{\prime}(r) q(r)\right| \leq C \frac{\varepsilon(\alpha)}{\alpha}
$$

for all $r \in \mathbb{R}$ and some constant $C$, which does not depend on $\alpha$. Finally, if $a_{\alpha}$ : $L^{2}(\Omega) \rightarrow L^{2}(\Omega), \quad a_{\alpha}(u)(x):=\beta_{\alpha}(u(x))$ denotes the superposition operator of $\beta_{\alpha}$, and $P_{H}: L^{2}(\Omega) \rightarrow H$ denotes the orthogonal projection of $L^{2}(\Omega)$ onto $H$, then it holds that $A_{\alpha}=P_{H} a_{\alpha}$.

Proof. 1. The definition of the Yosida approximation directly provides the formula for $\tilde{\beta}_{\alpha}$ and its derivative. Hence, by integration we obtain the relation for $\tilde{\gamma}_{\alpha}$.

2. The Lipschitz continuity and monotonicity of $\tilde{\beta}_{\alpha}$ and $\beta_{\alpha}$, respectively, are direct consequences of the properties of the Yosida approximation and the convolution with $\rho \geq 0$.

3. The inequality $\left|\beta_{\alpha}^{\prime}(r)\right| \leq \frac{1}{\alpha}$ is a consequence of the definition of $\beta_{\alpha}$ and the Lipschitz continuity of $\tilde{\beta}_{\alpha}$ with constant $\frac{1}{\alpha}$. In order to prove the second estimate of (4.1), we first notice that for an affine function $g(r):=c_{1} r+c_{2}$ it holds that $\left(g * \rho_{\varepsilon(\alpha)}\right)(r)=g(r)-c_{1} \varepsilon(\alpha) \bar{\rho}$, as readily seen by a simple calculation. This implies the assertion $\beta_{\alpha}(r)=\tilde{\beta}_{\alpha}(r)-\frac{\varepsilon(\alpha)}{\alpha} \bar{\rho}$ for $r \leq a-\varepsilon(\alpha)$ and $r \geq b+\varepsilon(\alpha)$ (note that outside the interval $[a-\varepsilon(\alpha), \beta+\varepsilon(\alpha)]$ the convolution $\tilde{\beta}_{\alpha} * \rho_{\varepsilon(\alpha)}$ only touches affine parts of $\left.\tilde{\beta}_{\alpha}\right)$. Since $\tilde{\beta}_{\alpha}$ and $\beta_{\alpha}$ are monotone, we conclude for $r \in[a-\varepsilon(\alpha), b+\varepsilon(\alpha)]$ 
that

$$
\begin{aligned}
\left|\beta_{\alpha}(r)-\tilde{\beta}_{\alpha}(r)\right| & \leq \max \left(\left|\tilde{\beta}_{\alpha}(b+\varepsilon(\alpha))-\beta_{\alpha}(a-\varepsilon(\alpha))\right|,\left|\beta_{\alpha}(b+\varepsilon(\alpha))-\tilde{\beta}_{\alpha}(a-\varepsilon(\alpha))\right|\right) \\
& \leq \frac{\varepsilon(\alpha)}{\alpha}(2+|\bar{\rho}|) .
\end{aligned}
$$

This also holds true for $r$ outside the interval. Now let us show the third inequality $\left|\beta_{\alpha}(r)-\beta_{\alpha}^{\prime}(r) q(r)\right| \leq C \frac{\varepsilon(\alpha)}{\alpha}$. Similar to $q$ we define

$$
q_{\alpha}(r):= \begin{cases}r-(a+2 \varepsilon(\alpha)) & \text { if } r \leq a+2 \varepsilon(\alpha), \\ 0 & \text { if } a+2 \varepsilon(\alpha)<r<b-2 \varepsilon(\alpha), \\ r-(b-2 \varepsilon(\alpha)) & \text { if } r \geq b-2 \varepsilon(\alpha)\end{cases}
$$

and assume that $\alpha$ is sufficiently small (such that $4 \varepsilon(\alpha)<b-a$ ). We have that $\left|\left(q-q_{\alpha}\right)(r)\right| \leq 2 \varepsilon(\alpha)$, and with $\sigma_{\varepsilon}(r):=r \rho_{\varepsilon}(r)$ we find

$$
\begin{aligned}
\left|\left(\tilde{\beta}_{\alpha}^{\prime} * \sigma_{\varepsilon(\alpha)}\right)(r)\right| & \leq \frac{1}{\alpha} \int_{\mathbb{R}}\left|\sigma_{\varepsilon(\alpha)}(r)\right| d r=\frac{1}{\alpha} \int_{\mathbb{R}}\left|\frac{r}{\varepsilon(\alpha)} \rho\left(\frac{r}{\varepsilon(\alpha)}\right)\right| d r \\
& =\frac{\varepsilon(\alpha)}{\alpha} \int_{\mathbb{R}}|s \rho(s)| d s=C \frac{\varepsilon(\alpha)}{\alpha} .
\end{aligned}
$$

Furthermore, for an affine function $g(r):=c_{1} r+c_{2}$ and any integrable function $f$, it holds that $\left[\left(f * \rho_{\varepsilon}\right) g\right](r)=\left[(g f) * \rho_{\varepsilon}\right](r)-\left(f * \sigma_{\varepsilon}\right)(r)$. Hence, we obtain

$$
\begin{aligned}
\left|\beta_{\alpha}(r)-\beta_{\alpha}^{\prime}(r) q(r)\right| & \leq\left|\beta_{\alpha}(r)-\beta_{\alpha}^{\prime}(r) q_{\alpha}(r)\right|+2 \frac{\varepsilon(\alpha)}{\alpha} \\
& =\left|\left[\tilde{\beta}_{\alpha} * \rho_{\varepsilon(\alpha)}-\left(q_{\alpha} \tilde{\beta}_{\alpha}^{\prime}\right) * \rho_{\varepsilon(\alpha)}\right](r)+\left(\tilde{\beta}_{\alpha}^{\prime} * \sigma_{\varepsilon(\alpha)}\right)(r)\right|+2 \frac{\varepsilon(\alpha)}{\alpha} \\
& \leq\left|\left[\left(\tilde{\beta}_{\alpha}-q \tilde{\beta}_{\alpha}^{\prime}\right) * \rho_{\varepsilon(\alpha)}\right](r)\right|+2 \frac{\varepsilon(\alpha)}{\alpha}+C \frac{\varepsilon(\alpha)}{\alpha}+2 \frac{\varepsilon(\alpha)}{\alpha} \\
& =(C+4) \frac{\varepsilon(\alpha)}{\alpha}
\end{aligned}
$$

due to $\tilde{\beta}_{\alpha}=q \tilde{\beta}_{\alpha}^{\prime}$ and the fact that the convolution $\beta_{\alpha}^{\prime} q_{\alpha}=\left(\tilde{\beta}_{\alpha}^{\prime} * \rho_{\varepsilon(\alpha)}\right) q_{\alpha}$ involves only affine parts of $q_{\alpha}$.

4. Finally, it is easy to show that for $\alpha>0$ we have

$$
\left\langle A_{\alpha} u, v\right\rangle_{V_{1}}=\int_{\Omega} v \beta_{\alpha}(u)
$$

for $u, v \in V_{1}$. Hence, $A_{\alpha}$ may be written as $P_{H} a_{\alpha}$.

Proposition 4.3. Assume the setting of Example 4.1 is fulfilled. Then there exists a constant $\alpha_{0}>0$ such that the functionals $\left(\varphi_{\alpha}\right)_{\alpha \in \bar{\Lambda}}, \varphi_{0}:=\varphi$, and $\psi$ satisfy Assumption 3.1.

Proof. We establish the statements (i)-(vi) of Assumption 3.1 step by step.

(i) The fact that $A_{\alpha}$ is single-valued is a consequences of Lemma 4.2.

(ii) From the properties of $\beta_{\alpha}$ given in Lemma 4.2 it follows that there are $0<$ $\alpha_{0}<1$ and $r_{0}>0$ such that $\gamma_{\alpha}(r) \geq \frac{1}{2} r^{2}$ and $r \beta_{\alpha}(r) \geq \frac{1}{2} r^{2}$ for all $0<\alpha \leq \alpha_{0}$ and 
$|r| \geq r_{0}$. Consequently, it holds that

$$
\begin{aligned}
\theta_{\alpha}(v)-\frac{1}{2}\|\cdot\|_{V_{1}}^{2} & \geq \varphi_{\alpha}(v)-\psi(v) \\
& \geq \int_{\left\{|v| \leq r_{0}\right\}} \gamma_{\alpha}(v)-\frac{1}{2}|v|^{2} d x+\int_{\left\{|v|>r_{0}\right\}} \gamma_{\alpha}(v)-\frac{1}{2}|v|^{2} d x \\
& \geq-\frac{r_{0}^{2}}{2}|\Omega| .
\end{aligned}
$$

This proves the desired boundedness from below.

(iii) Since $\beta_{\alpha} \in W^{1, \infty}(\Omega)$ it follows that $\beta_{\alpha} \circ u \in H^{1}(\Omega)$ for $u \in V_{1}$. Since $A_{\alpha}=P_{H} a_{\alpha}, A_{\alpha}$ can also be written as $A_{\alpha} u=P_{V}\left(\beta_{\alpha}^{\prime}(u)\right)$, where $P_{V}: H^{1}(\Omega) \rightarrow V_{1}$ denotes the orthogonal projection of $H^{1}(\Omega)$ onto $V_{1}$ with respect to the $H^{1}$-inner product. Consequently, we obtain $A_{\alpha}\left(\mathcal{V}_{1}\right) \subset \mathcal{V}_{1}$.

(iv) Next we show that $\widehat{\mathcal{A}}_{\alpha}: \mathcal{W}_{3} \rightarrow \mathcal{V}_{1}$ is continuously Fréchet differentiable. For this purpose, first notice that $\beta_{\alpha}$ is twice continuously differentiable with bounded first and second derivatives. Furthermore, by the Sobolev embedding theorem (cf. [5]) the space $V_{3}$ is continuously embedded into $W^{1, q_{1}}(\Omega)$ for arbitrary $q_{1}<\infty$ if $N \leq$ 4. This implies that $\mathcal{W}_{3}$ can be continuously embedded into $L^{p_{1}}\left(\mathcal{T} ; W^{1, q_{1}}(\Omega)\right)$ for $p_{1}=2$ as well as into $C\left(\overline{\mathcal{T}} ; V_{1}\right)$ by Proposition 2.9 , and hence in particular into $L^{p_{2}}\left(\mathcal{T} ; W^{1, q_{2}}(\Omega)\right)$ for $q_{2}=2$ and arbitrary $p_{2}<\infty$. Applying standard interpolation arguments (cf. Triebel [59]), the space $L^{p_{1}}\left(\mathcal{T} ; W^{1, q_{1}}(\Omega)\right) \cap L^{p_{2}}\left(\mathcal{T} ; W^{1, q_{2}}(\Omega)\right)$ can be embedded into $L^{p}\left(\mathcal{T} ; W^{1, q}(\Omega)\right)$ for all $1 \leq p, q<\infty$ with

$$
\frac{1}{p}=\frac{\theta}{p_{1}}+\frac{1-\theta}{p_{2}}, \quad \frac{1}{q}=\frac{\theta}{q_{1}}+\frac{1-\theta}{q_{2}}
$$

for $\theta \in] 0,1\left[\right.$. Let $\beta>1$ be given and set $\theta:=\frac{1}{1+\beta}, p_{2}:=\frac{4 \theta \beta}{1-2 \theta}$, and $q_{1}=\frac{p_{2}}{\beta^{2}}$. Then we obtain $1-\theta=\theta \beta$, and hence

$$
\frac{1}{p}=\frac{\theta}{2}+\frac{\theta \beta(1-2 \theta)}{4 \theta \beta}=\frac{1}{4}, \quad \frac{1}{q}=\frac{\theta \beta^{2}}{p_{2}}+\frac{\theta \beta}{2}=\beta\left(\frac{\theta}{2}+\frac{1-\theta}{p_{2}}\right)=\frac{\beta}{p} .
$$

Thus, $p=4$ and $q=\frac{p}{\beta}$. Since $\beta>1$ was arbitrary, Proposition 2.12 implies the continuous Fréchet differentiability of $\widehat{\mathcal{A}}_{\alpha}$.

Next, we prove that for each $y \in \mathcal{W}_{4}$ there exists an operator $\overline{D \mathcal{A}_{\alpha}}(y) \in \mathcal{L}\left(\mathcal{V}_{1} ; \mathcal{V}_{1}\right)$ with $\overline{D \mathcal{A}_{\alpha}}(y) v=D \widehat{\mathcal{A}}_{\alpha}(y) v$ for all $v \in \mathcal{W}_{3}$. This assertion is equivalent to saying that $D \widehat{\mathcal{A}}_{\alpha}(y) \in \mathcal{L}\left(\mathcal{W}_{3} ; \mathcal{V}_{1}\right)$ can be continuously extended to $\mathcal{V}_{1}$, or equivalently, that there exists a constant $C \geq 0$ with

$$
\left\|D \widehat{\mathcal{A}}_{\alpha}(y) v\right\|_{\mathcal{V}_{1}} \leq C\|v\|_{\mathcal{V}_{1}}
$$

for all $v_{1} \in \mathcal{W}_{3}$. Let us assume that $y \in \mathcal{W}_{4}$ and $v \in \mathcal{W}_{3}$. We have

$$
\left\|D \widehat{\mathcal{A}}_{\alpha}(y) v\right\|_{\mathcal{V}_{1}}^{2} \leq\left\|\nabla\left(\beta_{\alpha}^{\prime}(y) v\right)\right\|_{\mathcal{H}}^{2} \leq 2\left(\left\|\beta_{\alpha}^{\prime \prime}(y) v \nabla y\right\|_{\mathcal{H}}^{2}+\left\|\beta_{\alpha}^{\prime}(y) \nabla v\right\|_{\mathcal{H}}^{2}\right)
$$

and want to show that both terms on the right-hand side are bounded from above by $C\|v\|_{\mathcal{V}_{1}}$. Since $\beta_{\alpha}^{\prime}$ and $\beta_{\alpha}^{\prime \prime}$ have bounded range, it remains to be checked that $\|v \nabla y\|_{\mathcal{H}} \leq C\|v\|_{\mathcal{V}_{1}}$. For this purpose, notice that $\mathcal{W}_{4}$ can be continuously embedded into $C\left(\overline{\mathcal{T}} ; V_{2}\right)$; in particular $\nabla y \in L^{\infty}\left(\mathcal{T} ; H^{1}(\Omega)\right)$. By the Sobolev embedding theorem 
and since $N \leq 3, H^{1}(\Omega)$ embeds continuously into $L^{4}(\Omega)$. Therefore, it follows that

$$
\begin{aligned}
\|v \nabla y\|_{\mathcal{H}}^{2} & =\int_{\mathcal{T}}\|v \nabla y\|_{H}^{2} \leq \int_{\mathcal{T}}\|v\|_{L^{4}(\Omega)}^{2}\|\nabla y\|_{L^{4}(\Omega)}^{2} \\
& \leq C\|\nabla y\|_{L^{\infty}\left(\mathcal{T} ; L^{4}(\Omega)\right)}^{2}\|v\|_{L^{2}\left(\mathcal{T} ; L^{4}(\Omega)\right)}^{2} \\
& \leq C\|v\|_{\mathcal{V}_{1}}^{2} .
\end{aligned}
$$

(v) Let $\alpha \in \Lambda$ be given. Since $\mathcal{A}_{\alpha} u=P_{V}\left(\beta_{\alpha}(u)\right)$ and $0 \leq \beta_{\alpha}^{\prime}(r)$ for all $r \in \mathbb{R}$, we have that

$$
\left\langle\mathcal{L}_{1} y, \widehat{\mathcal{A}}_{\alpha} y\right\rangle_{\mathcal{V}_{1}}=\int_{\Omega} \nabla y \cdot \nabla\left(\beta_{\alpha}(y)\right)=\int_{\Omega} \beta_{\alpha}^{\prime}(y)|\nabla y|^{2} \geq 0 .
$$

(vi) Assuming $y_{n} \rightarrow y$ in $\mathcal{H}$, it follows from the Lipschitz continuity of $\beta_{\alpha}$ that $\mathcal{A}_{\alpha} y_{n}$ converges to $\mathcal{A}_{\alpha} y$ in $\mathcal{V}_{1}^{*}$. This proves (1).

In order to show (2), from $\left|\beta_{\alpha}(r)-\tilde{\beta}_{\alpha}(r)\right| \leq \frac{\varepsilon(\alpha)}{\alpha}(2+\bar{\rho})$ it follows that $\| \beta_{\alpha_{n}} \circ$ $y_{n}-\tilde{\beta}_{\alpha_{n}} \circ y_{n} \|_{\mathcal{H}} \leq C \frac{\varepsilon(\alpha)}{\alpha} \rightarrow 0$ as $\alpha \rightarrow 0$. Let $\widetilde{A}_{\alpha_{n}}: H \rightarrow H, \widetilde{A}_{\alpha_{n}} v:=\beta_{\alpha_{n}}(v)$ denote the Yosida approximation of $\partial \tilde{\gamma}$ with parameter $\alpha_{n}$. Consequently, it holds that $\widetilde{\mathcal{A}}_{\alpha_{n}} y_{n} \rightarrow h$ in $\mathcal{H}$. Since $\partial \tilde{\gamma}$ is maximal-monotone, we invoke Proposition 2.5 of [20] in order to conclude $(y, h) \in \partial \tilde{\gamma}$ and hence $(y, h) \in \mathcal{A}$.

Let us choose $\alpha_{0}>0$ so small that $\varepsilon(\alpha)<\min \left\{\inf _{\Omega} y_{0}-a, b-\sup _{\Omega} y_{0}\right\}$ for all $\alpha \in$ $\left[0, \alpha_{0}\right]$. Then $\beta_{\alpha}\left(y_{0}(x)\right)=0$, and $y_{0}$ satisfies the conditions given in Assumption 3.1. This completes the proof.

Remark 4.4. For $\alpha$ sufficiently small, $\beta_{\alpha}$ vanishes identically in a neighborhood of 0 . We could choose different mollifiers $\rho^{1}$ and $\rho^{2}$ instead of $\rho$ in the definition of $\beta_{\alpha}(r)$ for either positive $r$ or negative $r$, respectively. Thus, the assertion of Proposition 4.3 remains true also in this case.

CONVEntion 4.5. In the last theorem below we will use projection and superposition operators which do not preserve the mean value. In order to simplify the notation, we extend the inner product of $H$ to a semi-inner product on $L^{2}(\Omega)$ by

$$
(u \mid v)_{H}:=(Q u \mid Q v)_{H}
$$

for $u, v \in L^{2}(\Omega)$, where $Q: L^{2}(\Omega) \rightarrow H$ denotes the orthogonal projection of $L^{2}(Q)$ onto $H$. Likewise, we define

$$
\langle u, v\rangle_{V_{1}}:=\left\langle\left. u\right|_{V_{1}}, Q v\right\rangle_{V_{1}}
$$

for $u \in\left(H^{1}(\Omega)\right)^{*}, v \in H^{1}(\Omega)$. This is well defined since $Q$ maps $H^{1}(\Omega)$ onto $V_{1}$. Accordingly, the inner product of $\mathcal{H}$ and the dual pairing between $\mathcal{V}_{1}$ and its dual are extended to $L^{2}\left(\mathcal{T} ; L^{1}(\Omega)\right)$, respectively, $L^{2}\left(\mathcal{T} ; H^{1}(\Omega)\right)$ and its dual.

Finally, for the double-obstacle potential according to Example 4.1 we study further properties of various dual quantities involved in the system established in Theorem 3.16. This corresponds to a function space version of C-stationarity; cf. [41, 57].

THEOREM 4.6. Let the setting of Example 4.1 and the assumptions of Theorem 3.16 be satisfied. Using the notation $\xi_{n}:=\widehat{\mathcal{A}}_{\alpha_{n}} y_{n}$ and $\lambda_{n}:=\overline{D \mathcal{A}_{\alpha_{n}}}\left(y_{n}\right)^{*} \mathcal{L}_{3} p_{n}$ and the results of Theorem 3.16, it follows that

$$
\begin{gathered}
y^{\prime}+\mathcal{L}_{1} w=u, \quad w=\mathcal{L}_{1} y+\xi+\eta \mathcal{I}_{1} y, \\
-p^{\prime}+\mathcal{L}_{1} \mathcal{L}_{3} p+\lambda+\eta \mathcal{I}_{1} \mathcal{L}_{3} p=\mu_{1}\left(y-y_{\Omega}\right), \\
p+u \in\left(\mathbb{R}^{+}(\mathcal{C}-u)\right)^{+}
\end{gathered}
$$


If furthermore $\left(\lambda_{m}\right)$ is bounded in $\mathcal{V}_{1}^{*}$ and $h: \mathbb{R} \rightarrow \mathbb{R}$ is a Lipschitz function which satisfies $h(a)=h(b)=0$, then the following relations hold true:

$$
\begin{aligned}
& (\xi \mid y-a)_{\mathcal{H}}=0,(\xi \mid y-b)_{\mathcal{H}}=0, \\
& \lim \left(\lambda_{m} \mid h\left(y_{m}\right)\right)_{\mathcal{H}}=0, \lim \left(\xi_{m} \mid \mathcal{L}_{3} p_{m}\right)_{\mathcal{H}}=0, \\
& \underline{\lim }\left(\lambda_{m} \mid \mathcal{L}_{3} p_{m}\right)_{\mathcal{H}} \geq 0, \\
& \lambda_{k} \rightarrow 0 \quad \text { almost everywhere on }\{z \in \mathcal{T} \times \Omega: a<y(z)<b\}
\end{aligned}
$$

for a subsequence $\left(\lambda_{k}\right)$ of $\left(\lambda_{m}\right)$.

Proof. 1. We start by showing the complementarity conditions $(\xi \mid y-a)_{\mathcal{H}}=$ $0,(\xi \mid y-b)_{\mathcal{H}}=0$. For this purpose, recall that Assumption 3.1 ensures that $(y, \xi) \in \mathcal{A}$ and that $\mathcal{A}$ is the superposition operator of the subdifferential of $\varphi=\imath_{K_{V}}: V_{1} \rightarrow \overline{\mathbb{R}}$. Since $\xi \in \mathcal{H}$, we therefore may conclude that $(y(z), \xi(z)) \in \partial \imath_{M}$ for almost all $z \in$ $\overline{\mathcal{T}} \times \Omega$, which implies $\xi(z)(y(z)-a)=0$ and $\xi(z)(y(z)-b)=0$. Integration yields the desired complementarity conditions.

2. Next, we prove $\lim \left(\lambda_{m} \mid h\left(y_{m}\right)\right)_{\mathcal{H}}=0$. Denoting the metric projection of $\mathbb{R}$ onto $M=[a, b]$ by $p_{M}$, the metric projection of $H$ onto $K_{H}$ by $P$ (which is the superposition operator of $p_{M}$ ), and the superposition operator of $P$ with respect to $\mathcal{T}$ by $\mathcal{P}$, and taking advantage of the continuity of the superposition operator of $h$ on $V_{1}$ (cf. [48]), it follows that $\mathcal{P}\left(\mathcal{V}_{1}\right) \subset \mathcal{V}_{1}$ and $\lim \mathcal{P} y_{m}=\mathcal{P} y=y, \lim h\left(\mathcal{P} y_{m}\right)=$ $h(\mathcal{P} y)=h(y)=\lim h\left(y_{m}\right)$ in $L^{2}\left(\mathcal{T} ; H^{1}(\Omega)\right)$. We know that $\left|\beta_{\alpha}^{\prime}(r)\right| \leq \frac{1}{\alpha}$ for all $r$ and $\beta_{\alpha}^{\prime}(r)=0$ for $a+\varepsilon(\alpha) \leq r \leq b-\varepsilon(\alpha)$. If $L_{h}$ is the Lipschitz constant of $h$, then $|h(r)| \leq L_{h} \min (|r-a|,|r-b|)$ for $r \in \mathbb{R}$. Consequently, it follows that

$$
\begin{aligned}
\left|\left(\lambda_{m} \mid h\left(\mathcal{P} y_{m}\right)\right)_{\mathcal{H}}\right|^{2} & =\left|\left(\mathcal{L}_{3} p_{m} \mid \overline{D \mathcal{A}_{\alpha_{m}}}\left(y_{m}\right) h\left(\mathcal{P} y_{m}\right)\right)_{\mathcal{H}}\right|^{2} \\
& \leq|| \mathcal{L}_{3} p_{m} \|_{\mathcal{H}}^{2} \int_{\mathcal{T} \times \Omega}\left|\beta_{\alpha_{m}}^{\prime}\left(y_{m}\right) h\left(\mathcal{P} y_{m}\right)\right|^{2} \\
& \leq\left(|\mathcal{T} \times \Omega|\left\|\mathcal{L}_{3} p_{m}\right\|_{\mathcal{H}} L_{h} \frac{\varepsilon\left(\alpha_{m}\right)}{\alpha_{m}}\right)^{2} \rightarrow 0
\end{aligned}
$$

as $m \rightarrow \infty$. Moreover, since $\left(\lambda_{m}\right)$ is bounded in $\mathcal{V}_{1}^{*}$ we have that

$\lim \left(\lambda_{m} \mid h\left(y_{m}\right)\right)_{\mathcal{H}}=\lim \left(\lambda_{m} \mid h\left(\mathcal{P} y_{m}\right)\right)_{\mathcal{H}}+\lim \left\langle\lambda_{m}, h\left(y_{m}\right)-h\left(\mathcal{P} y_{m}\right)\right\rangle_{\mathcal{V}_{1}}=0$.

3. We set $g_{m}(r):=\beta_{\alpha_{m}}(r)-\beta_{\alpha_{m}}^{\prime}(r) q(r)$. With $r-p_{M}(r)=q(r)$ we find

$$
\begin{aligned}
\left(\xi_{m} \mid \mathcal{L}_{3} p_{m}\right)_{\mathcal{H}} & =\left(\mathcal{L}_{3} p_{m} \mid \beta_{\alpha_{m}}\left(y_{m}\right)\right)_{\mathcal{H}} \\
& =\left(\mathcal{L}_{3} p_{m} \mid g_{m}\left(y_{m}\right)\right)_{\mathcal{H}}+\left(\lambda_{m} \mid y_{m}-\mathcal{P} y_{m}\right)_{\mathcal{H}}
\end{aligned}
$$

By Lemma 4.2, for $m$ sufficiently large it holds that $\left|g_{m}(r)\right|=\left|\beta_{\alpha_{m}}(r)-\beta_{\alpha_{m}}^{\prime}(r) q(r)\right| \leq$ $C \frac{\varepsilon\left(\alpha_{m}\right)}{\alpha_{m}}$. Hence, the first term on the right-hand side converges to 0 . So does the second, since $\left(\lambda_{m}\right)$ is bounded in $\mathcal{V}_{1}^{*}$ and since $\left(y_{m}\right)$ and $\left(\mathcal{P} y_{m}\right)$ both converge to $y$ in $\mathcal{V}_{1}$.

4. The fact that $\underline{\lim }\left\langle\lambda_{m}, \mathcal{L}_{3} p_{m}\right\rangle_{\mathcal{V}_{1}} \geq 0$ is an obvious consequence of Lemma 3.13.

5. Let us fix representatives of the equivalence classes $y,\left(y_{m}\right)$ and denote $Z$ the set $\{z \in \mathcal{T} \times \Omega: a<y(z)<b\}$. Since $y_{m}$ converges to $y$ in $\mathcal{V}_{1}$, a subsequence $\left(y_{k}\right)$ of $\left(y_{m}\right)$ converges almost everywhere on $\mathcal{T} \times \Omega$ to $y$. Moreover, we know that $\varepsilon\left(\alpha_{m}\right) \rightarrow 0$. 
Hence, for almost all $z \in Z$ there exists $k_{0}(z)$ such that $a+\varepsilon\left(\alpha_{k}\right)<y_{k}(z)<b-\varepsilon\left(\alpha_{k}\right)$ for all $k \geq k_{0}(z)$.

From the properties of $\beta_{\alpha}$ it therefore follows that $\lambda_{k}(z)=0$ for almost all $z \in Z$ and $k \geq k_{0}(z)$. Consequently, $\lambda_{k}$ converges to 0 almost everywhere on $Z$.

Combining the results of Theorems 3.16 and 4.6 and considering the sign condition satisfied by $\lambda_{m}$ and $p_{m}$ in the limit, we find that our stationarity system corresponds to a function space version of C-stationarity for MPECs; see [41, 43, 57].

We end this section by briefly discussing the relevance of C-stationarity in theory and numerical practice. Compared to weaker forms of stationarity, for instance, those contained in [11], C-stationarity represents a sharper stationarity notion avoiding spurious stationarity points. On the numerical level, it appears possible to extend the algorithms in [43] to the Cahn-Hilliard setting, which would then yield C-stationary points of the associated discrete problems.

Appendix A. Proofs for section 2. In this appendix we provide proofs of results given in section 2. We start with the basic Lemma 2.4.

Proof of Lemma 2.4. Let $\left(y, y^{*}\right) \in Y \times Y^{*}$ be given and define $\psi:=\operatorname{Ext}(\varphi, X, Y, E)$. Then we have that

$$
\begin{aligned}
\left(y, y^{*}\right) & \in \partial \psi \\
& \Longleftrightarrow y \in \operatorname{dom} \psi,\left\langle y^{*}, v-y\right\rangle_{Y} \leq \psi(v)-\psi(y) \quad \forall v \in Y \\
& \Longleftrightarrow y \in \operatorname{dom} \psi, \quad u:=E^{-1} y, \quad\left\langle E^{*} y^{*}, x-u\right\rangle_{X} \leq \varphi(x)-\varphi(u) \quad \forall x \in X \\
& \Longleftrightarrow\left(E^{-1} y, E^{*} y^{*}\right) \in \partial \varphi .
\end{aligned}
$$

This implies $\partial \psi=\left(E^{*}\right)^{-1} \partial \varphi E^{-1}$. Now assume that $\varphi$ is proper, convex, and lowersemicontinuous and has bounded lower-level sets. It is clear that $\psi$ is proper and convex. Let $\left(y_{n}\right)$ be a sequence in $Y$ that converges strongly to $y \in Y$. We have to show that $\psi(y) \leq \underline{\lim } \psi\left(y_{n}\right)$.

If $\underline{\lim } \psi\left(y_{n}\right)=\infty$, then the assertion is trivial. So let us assume that $\underline{\lim } \psi\left(y_{n}\right)<$ $\infty$. Hence, it is possible to extract a subsequence $\left(y_{m}\right)$ of $\left(y_{n}\right)$ such that $y_{m} \in \operatorname{dom} \psi$ and $\underline{\lim } \psi\left(y_{n}\right)=\lim \psi\left(y_{m}\right)$. We set $x_{m}:=E^{-1} y_{m}$, and since $\left(\varphi\left(x_{m}\right)\right)$ is bounded in $\mathbb{R},\left(x_{m}\right)$ has to be bounded in $X$. Consequently, there exists a subsequence $\left(x_{k}\right)$ of $\left(x_{m}\right)$ that converges weakly in $X$. The continuity and injectivity of $E$ therefore imply that this weak limit is $E^{-1} y$. Since $\varphi$ is convex and lower-semicontinuous, it is even weakly lower-semicontinuous. This implies

$$
\psi(y)=\varphi\left(E^{-1} y\right) \leq \varliminf_{k \rightarrow \infty} \varphi\left(x_{k}\right)=\varliminf_{n \rightarrow \infty} \psi\left(y_{n}\right)
$$

and therefore finishes the proof.

Next, the commutation rules and the regularity result for the bi-Laplace equations are established.

Proof of Proposition 2.8. Since $\partial \Omega$ is supposed to be sufficiently smooth, standard regularity results imply that $L_{3}: V_{3} \rightarrow V_{1}$ is an isomorphism and that $I_{1} L_{3}=L_{1} I_{3}$ (cf. [35]). Furthermore, the unitarity of $L_{1}$ and $L_{3}$ is easily verified. Now let $v_{0} \in V_{3}$ and $v_{1} \in V_{1}$ be given. From the symmetry of $I_{1}$ and $L_{1}$ it follows that

$$
\begin{aligned}
\left\langle I_{3}^{*} L_{1} v_{1}, v_{0}\right\rangle_{V_{3}} & =\left\langle L_{1} v_{1}, I_{3} v_{0}\right\rangle_{V_{1}}=\left\langle L_{1} I_{3} v_{0}, v_{1}\right\rangle_{V_{1}}=\left\langle I_{1} L_{3} v_{0}, v_{1}\right\rangle_{V_{1}}=\left\langle I_{1} v_{1}, L_{3} v_{0}\right\rangle_{V_{1}} \\
& =\left\langle L_{3}^{*} I_{1} v_{1}, v_{0}\right\rangle_{V_{3}} .
\end{aligned}
$$


Thus, we have that $I_{3}^{*} L_{1}=L_{3}^{*} I_{1}$. Consider $y, v \in V_{1}$ and $y^{*}, v^{*} \in V_{1}^{*}$. Since

$$
\begin{gathered}
\left(L_{1}^{-1} I_{1} y \mid v\right)_{V_{1}}=\left\langle I_{1} y, v\right\rangle_{V_{1}}, \\
\left(\left(L_{3}^{*}\right)^{-1} I_{3}^{*} y^{*} \mid v^{*}\right)_{V_{1}^{*}}=\left(I_{1} L_{1}^{-1} y^{*} \mid L_{1} L_{1}^{-1} v^{*}\right)_{V_{1}^{*}}=\left\langle I_{1} L_{1}^{-1} y^{*}, L_{1}^{-1} v^{*}\right\rangle_{V_{1}},
\end{gathered}
$$

the symmetry, positivity, and injectivity of $L_{1}^{-1} I_{1}$ and $\left(L_{3}^{*}\right)^{-1} I_{3}^{*}$ are obvious. By Rellich's lemma, $I_{V_{1}}$ is compact and therefore also are $I_{1}$ and $L_{1}^{-1} I_{1}$. The same applies to $I_{3}$ and $\left(L_{3}^{*}\right)^{-1} I_{3}^{*}$ by the Sobolev embedding theorem.

Assume that $U_{1}$ is a closed subspace of $V_{1}$ satisfying $L_{1}^{-1} I_{1}\left(U_{1}\right)=U_{1}$. The continuity of $L_{1}^{-1}$ implies that $I_{1}\left(U_{1}\right)$ is closed in $V_{1}^{*}$ as well. Since $U_{1}$ is a Hilbert space with the induced inner product of $V_{1}$, we conclude from the spectral theory of self-adjoint, compact operators the existence of a finite or countable sequence of orthonormal eigenvectors $\left(v_{n}\right)_{n \in N}$ with $N \subset \mathbb{N}$ in $U_{1}$ of $L_{1}^{-1} I_{1}$ for the eigenvalues $\left(\lambda_{n}\right)_{n \in N}$ such that $U_{1}=\bigoplus_{n \in N} \mathbb{R} v_{n}$. Moreover, it holds that $\lambda_{n}>0$ for all $n \in N$ and $\lambda_{n} \rightarrow 0$ for $n \rightarrow \infty, n \in N$. Since $L_{1}: V_{1} \rightarrow V_{1}^{*}$ is a unitary operator and $I_{1}\left(U_{1}\right)=L_{1}\left(U_{1}\right)$, the orthogonal projections $P_{V_{1} \rightarrow U_{1}}$ and $P_{V_{1}^{*} \rightarrow I_{1}\left(U_{1}\right)}$ from $V_{1}$ onto $U_{1}$ and from $V_{1}^{*}$ onto $I_{1}\left(U_{1}\right)$, respectively, are given by

$$
P_{V_{1} \rightarrow U_{1}} v=\sum_{n \in N}\left(v \mid v_{n}\right)_{V_{1}} v_{n} \quad \text { and } \quad P_{V_{1}^{*} \rightarrow I_{1}\left(U_{1}\right)} v^{*}=\sum_{n \in N}\left(v^{*} \mid L_{1} v_{n}\right)_{V_{1}^{*}} L_{1} v_{n} .
$$

We have $L_{1}^{-1} I_{1} v_{n}=\lambda_{n} v_{n}$, and hence $\lambda_{n} L_{1} v_{n}=I_{1} v_{n}$. Furthermore, it holds that

$$
\left(I_{1} v \mid L_{1} v_{n}\right)_{V_{1}^{*}}=\left\langle I_{1} v, v_{n}\right\rangle_{V_{1}}=\left\langle I_{1} v_{n}, v\right\rangle_{V_{1}}=\lambda_{n}\left\langle L_{1} v_{n}, v\right\rangle_{V_{1}}=\lambda_{n}\left(v_{n} \mid v\right)_{V_{1}} .
$$

Altogether, this yields

$$
\begin{aligned}
I_{1} P_{V_{1} \rightarrow U_{1}} v & =I_{1}\left(\sum_{n \in N}\left(v \mid v_{n}\right)_{V_{1}} v_{n}\right)=\sum_{n \in N}\left(v \mid v_{n}\right)_{V_{1}} I_{1} v_{n}, \\
P_{V_{1}^{*} \rightarrow I_{1}\left(U_{1}\right)} I_{1} v & =\sum_{n \in N}\left(I_{1} v \mid L_{1} v_{n}\right)_{V_{1}^{*}} L_{1} v_{n}=\sum_{n \in N} \lambda_{n}\left(v \mid v_{n}\right)_{V_{1}} \frac{1}{\lambda_{n}} I_{1} v_{n}, \\
L_{1} P_{V_{1} \rightarrow U_{1}} v & =L_{1}\left(\sum_{n \in N}\left(v \mid v_{n}\right)_{V_{1}} v_{n}\right)=\sum_{n \in N}\left(v \mid v_{n}\right)_{V_{1}} L_{1} v_{n}, \\
P_{V_{1}^{*} \rightarrow I_{1}\left(U_{1}\right)} L_{1} v & =\sum_{n \in N}\left(L_{1} v \mid L_{1} v_{n}\right)_{V_{1}^{*}} L_{1} v_{n}=\sum_{n \in N}\left(v \mid v_{n}\right)_{V_{1}} L_{1} v_{n},
\end{aligned}
$$

and therefore $I_{1} P_{V_{1} \rightarrow U_{1}}=P_{V_{1}^{*} \rightarrow I_{1}\left(U_{1}\right)} I_{1}$ and $L_{1} P_{V_{1} \rightarrow U_{1}}=P_{V_{1}^{*} \rightarrow I_{1}\left(U_{1}\right)} L_{1}$. Similar arguments can be used to prove $I_{3}^{*} P_{V_{1}^{*} \rightarrow U_{1}^{*}}=P_{V_{3}^{*} \rightarrow I_{3}^{*}\left(U_{1}^{*}\right)} I_{3}^{*}$ and $L_{3}^{*} P_{V_{1}^{*} \rightarrow U_{1}^{*}}=$ $P_{V_{3}^{*} \rightarrow I_{3}^{*}\left(U_{1}^{*}\right)} L_{3}^{*}$. cf. [61].

The following general integration by parts formula is a result of Gröger [36];

Proposition A.1. Let $V$ be a reflexive Banach space, let $H$ be an arbitrary Hilbert space, and let $K \in \mathcal{L}(V ; H)$ with dense range. We define $E:=K^{*} J_{H} K \in$ $\mathcal{L}\left(V ; V^{*}\right)$ and $\mathcal{H}:=L^{2}(\mathcal{T} ; H), \mathcal{V}:=L^{2}(\mathcal{T} ; V), \mathcal{W}:=\left\{y \in \mathcal{V}:(\mathcal{E} y)^{\prime} \in \mathcal{V}^{*}\right\}$ with the standard norms and $\|y\|_{\mathcal{W}}:=\left(\|y\|_{\mathcal{V}}^{2}+\left\|(\mathcal{E} y)^{\prime}\right\|_{\mathcal{V}^{*}}^{2}\right)^{1 / 2}$, respectively. Then the operator $\mathcal{K}$ maps $\mathcal{W}$ continuously into the space $C(\overline{\mathcal{T}} ; H)$, meaning that every class of equivalent 
functions in $\mathcal{K}(\mathcal{W}) \subset L^{2}(\mathcal{T} ; H)$ possesses a representative that is continuous from $\mathcal{T}$ into $H$ with continuous extension onto $\overline{\mathcal{T}}$. Furthermore, in this sense the formulas

$$
\begin{aligned}
& \left((\mathcal{K} y)\left(t_{2}\right) \mid(\mathcal{K} v)\left(t_{2}\right)\right)_{H}-\left((\mathcal{K} y)\left(t_{1}\right) \mid(\mathcal{K} v)\left(t_{1}\right)\right)_{H} \\
& =\int_{t_{1}}^{t_{2}}\left[\left\langle(\mathcal{E} y)^{\prime}(t), v(t)\right\rangle_{V}+\left\langle(\mathcal{E} v)^{\prime}(t), y(t)\right\rangle_{V}\right] d t,
\end{aligned}
$$

and in particular

$$
\left\|(\mathcal{K} y)\left(t_{2}\right)\right\|_{H}^{2}-\left\|(\mathcal{K} y)\left(t_{1}\right)\right\|_{H}^{2}=2 \int_{t_{1}}^{t_{2}}\left\langle(\mathcal{E} y)^{\prime}(t), y(t)\right\rangle_{V} d t,
$$

hold for all $y, v \in \mathcal{W}$ and $t_{1}, t_{2} \in \overline{\mathcal{T}}$.

Proof. 1. First, assume that $y, v \in C_{c}^{1}(\mathbb{R} ; V)$. Let us define the functional $\varphi(t):=((\mathcal{K} y)(t) \mid(\mathcal{K} v)(t))_{H}=(K y(t) \mid K v(t))_{H}$. It is easy to see that $\varphi \in C^{1}(\mathbb{R})$ and

$\varphi^{\prime}(t)=\left(K y^{\prime}(t) \mid K v(t)\right)_{H}+\left(K v^{\prime}(t) \mid K y(t)\right)_{H}=\left\langle(\mathcal{E} y)^{\prime}(t), v(t)\right\rangle_{V}+\left\langle(\mathcal{E} v)^{\prime}(t), y(t)\right\rangle_{V}$.

Hence, (A.1) and (A.2) follow from the fundamental theorem of calculus.

2. Again, let us suppose that $y \in C_{c}^{1}(\mathbb{R} ; V)$ and $t, s \in \mathbb{R}$. By virtue of (A.2) and Hölder's inequality, we conclude that

$$
\|(\mathcal{K} y)(t)\|_{H}^{2} \leq\|(\mathcal{K} y)(s)\|_{H}^{2}+2\left\|(\mathcal{E} y)^{\prime}\right\|_{\mathcal{V}^{*}}\|y\|_{\mathcal{V}} \leq\|(\mathcal{K} y)(s)\|_{H}^{2}+2\|y\|_{\mathcal{W}}^{2} .
$$

After applying the square root on both sides and integrating over $s \in \operatorname{supp} y$, we can estimate

$$
|\operatorname{supp} y|\|(\mathcal{K} y)(t)\|_{H} \leq\|\mathcal{K} y\|_{L^{1}(\mathbb{R} ; H)}+\sqrt{2}|\operatorname{supp} y|\|y\|_{\mathcal{W}} .
$$

Assume that $|\operatorname{supp} y|>0$. Since $\mathcal{K} \in L\left(L^{2}(\mathbb{R} ; V) ; L^{2}(\mathbb{R} ; V)\right)$, we obtain

$$
\sup _{t \in \mathbb{R}}\|(\mathcal{K} y)(t)\|_{H} \leq C\left(\|y\|_{\mathcal{V}}+\|y\|_{\mathcal{W}}\right) \leq C\|y\|_{\mathcal{W}}
$$

for constant $C$ depending only on $|\operatorname{supp} y|$. If $|\operatorname{supp} y|=0$, this inequality obviously holds as well.

3. Let $y \in \mathcal{W}$ be arbitrary. Since the restriction to $\mathcal{T}$ of the set of continuously differentiable functions on $\mathbb{R}$ with values in $V$ is dense in $\mathcal{W}$, there exists a sequence $\left(y_{n}\right)$ in $C_{c}^{1}(\mathbb{R} ; V)$ such that $\left.y_{n}\right|_{\mathcal{T}} \rightarrow y$ in $\mathcal{W}$. From (A.3) it follows that $\mathcal{K} y_{n}$ is a Cauchy sequence in $C(\overline{\mathcal{T}} ; H)$ and therefore converges to some $h \in C(\overline{\mathcal{T}} ; H)$. It is not difficult to see that $\mathcal{K} y=h$ almost everywhere on $\mathcal{T}$. Hence, $h$ is the continuous representative of $\mathcal{K} y \in L^{\infty}(\overline{\mathcal{T}} ; H)$. Moreover, in the estimation in steps 1 and 2 we can pass to the limit in order to obtain the assertion for arbitrary $y, v \in \mathcal{W}$. This completes the proof.

Proof of Proposition 2.9. From Proposition A.1 and with $L_{1}=J_{V}$ we obtain that for functions $f, g \in \mathcal{V}_{3}$ with $\mathcal{E} f, \mathcal{E} g \in H^{1}\left(\mathcal{T} ; V_{3}^{*}\right)$ for $E=I_{3}^{*} L_{1} I_{3}$ it holds that $f, g$ admit continuous representatives defined on $\overline{\mathcal{T}}$ with values in $V_{1}$ and

$$
\left\langle(\mathcal{E} f)^{\prime}, g\right\rangle_{\mathcal{V}_{3}}+\left\langle(\mathcal{E} g)^{\prime}, f\right\rangle_{\mathcal{V}_{3}}=(f(T) \mid g(T))_{V_{1}}-(f(0) \mid g(0))_{V_{1}} .
$$

Proposition 2.8 implies

$$
E=I_{3}^{*} L_{1} I_{3}=L_{3}^{*} I_{1} I_{3}=I_{3}^{*} I_{1} L_{3} .
$$


For $y, v$ as in the assertion, let us choose $f:=y \in \mathcal{V}_{3}$ and $g:=L_{3}^{-1} v \in \mathcal{V}_{3}$. Thus, we have $H^{1}\left(\mathcal{T} ; V_{1}^{*}\right) \ni y=\left(\mathcal{L}_{3}^{*}\right)^{-1} \mathcal{E} f$ and $H^{1}\left(\mathcal{T} ; V_{3}^{*}\right) \ni v=\mathcal{E} g$ and therefore $\mathcal{E} f, \mathcal{E} g \in$ $H^{1}\left(\mathcal{T} ; V_{3}^{*}\right)$. This implies that $f=y$ and $g=\mathcal{L}_{1}^{-1} v$ can be regarded as continuous functions on $\overline{\mathcal{T}}$ with values in $V_{1}$ (and hence also $v$ with values in $V_{1}^{*}$ ). Moreover, it follows that

$$
\begin{aligned}
\left\langle y^{\prime}, v\right\rangle_{\mathcal{V}_{1}} & +\left\langle v^{\prime}, y\right\rangle_{\mathcal{V}_{3}} \\
& =\left\langle\left(\mathcal{L}_{3}^{*}\right)^{-1}(\mathcal{E} f)^{\prime}, \mathcal{L}_{3} g\right\rangle_{\mathcal{V}_{1}}+\left\langle(\mathcal{E} g)^{\prime}, f\right\rangle_{\mathcal{V}_{3}}=\left\langle(\mathcal{E} f)^{\prime}, g\right\rangle_{\mathcal{V}_{3}}+\left\langle(\mathcal{E} g)^{\prime}, f\right\rangle_{\mathcal{V}_{3}} \\
& =(f(T) \mid g(T))_{V_{1}}-(f(0) \mid g(0))_{V_{1}} \\
& =\left\langle\left(\mathcal{L}_{1} g\right)(T), f(T)\right\rangle_{V_{1}}-\left\langle\left(\mathcal{L}_{1} g\right)(0), f(0)\right\rangle_{V_{1}} \\
& =\langle v(T), y(T)\rangle_{V_{1}}-\langle v(T), y(0)\rangle_{V_{1}} .
\end{aligned}
$$

This completes the proof.

Proof of Theorem 2.10. Choose a complete orthonormal system of eigenvectors $\left(e_{n}\right)_{n \in \mathbb{N}}$ for the eigenvalues $\left(\lambda_{n}\right)_{n \in \mathbb{N}}$ in $V_{1}$ of the operator $L_{1}^{-1} I_{1}$. We denote the span of $e_{1}, \ldots, e_{n}$ by $V_{1, n}$ and $V_{1, n}^{*}:=I_{1}\left(V_{1, n}\right) \subset V_{1}^{*}, V_{3, n}^{*}:=I_{3}^{*}\left(V_{1, n}^{*}\right) \subset V_{3}^{*}$. The orthogonal projection of $V_{1}, V_{1}^{*}$, and $V_{3}^{*}$ onto the subspaces $V_{1, n}, V_{1, n}^{*}$, and $V_{3, n}^{*}$ are denoted by $P_{V_{1, n}}, P_{V_{1, n}^{*}}$, and $P_{V_{3, n}^{*}}$, respectively. Then it is not hard to show that $P_{V_{1, n}} y=: y_{n} \rightarrow y$ in $\mathcal{V}_{1}, y_{n} \in H^{1}\left(\mathcal{T} ; V_{1, n}\right)$, and

$$
\mathcal{P}_{V_{3, n}^{*}} y^{\prime}=y_{n}^{\prime} \rightarrow y^{\prime} \quad \text { in } \mathcal{V}_{3}^{*} .
$$

Moreover, the continuity of $L_{3}$ and $L_{1}$ implies that $\mathcal{L}_{3}^{*} \mathcal{L}_{1} y_{n} \rightarrow \mathcal{L}_{3}^{*} \mathcal{L}_{1} y$ in $\mathcal{V}_{3}^{*}$. With $\lambda_{n} e_{n}=L_{1}^{-1} I_{1} e_{n}=I_{3} L_{3}^{-1} e_{n}$, it holds that $e_{n} \in V_{3}$. Hence $y_{n} \in L^{2}\left(\mathcal{T} ; V_{3}\right)$.

In order to prove the assertion, it suffices to show that $\left(y_{n}\right)$ and $\left(y_{n}^{\prime}\right)$ remain bounded in $\mathcal{V}_{3}$ and $\mathcal{V}_{1}^{*}$, respectively. From $y^{\prime}+\mathcal{L}_{3}^{*} \mathcal{L}_{1} y=f$ and Proposition 2.8 we obtain

$$
y_{n}^{\prime}+\mathcal{L}_{1} \mathcal{L}_{3} y_{n}=\mathcal{P}_{V_{3, n}^{*}}\left[y^{\prime}+\mathcal{L}_{3}^{*} \mathcal{L}_{1} y\right]=\mathcal{P}_{V_{1, n}^{*}} f,
$$

and therefore using $\|f\|_{\mathcal{V}_{1}^{*}} \geq\left\|\mathcal{P}_{V_{1, n}^{*}} f\right\|_{\mathcal{V}_{1}^{*}}$ also, we obtain

$$
\|f\|_{\mathcal{V}_{1}^{*}}^{2} \geq\left\|y_{n}^{\prime}+\mathcal{L}_{1} \mathcal{L}_{3} y_{n}\right\|_{\mathcal{V}_{1}^{*}}^{2}=\left\|y_{n}^{\prime}\right\|_{\mathcal{V}_{1}^{*}}^{2}+2\left(y_{n}^{\prime} \mid \mathcal{L}_{1} \mathcal{L}_{3} y_{n}\right)_{\mathcal{V}_{1}^{*}}+\left\|\mathcal{L}_{1} \mathcal{L}_{3} y_{n}\right\|_{\mathcal{V}_{1}^{*}}^{2} .
$$

The term on the right-hand side can be transformed into

$$
\begin{aligned}
\left(y_{n}^{\prime} \mid \mathcal{L}_{1} \mathcal{L}_{3} y_{n}\right)_{\mathcal{V}_{1}^{*}} & =\left\langle y_{n}^{\prime}, \mathcal{L}_{3} y_{n}\right\rangle_{\mathcal{V}_{1}}=\left\langle\mathcal{L}_{1} y_{n}, y_{n}^{\prime}\right\rangle_{\mathcal{V}_{1}}=\left(y_{n} \mid y_{n}^{\prime}\right)_{\mathcal{V}_{1}} \\
& =\frac{1}{2}\left(\left\|y_{n}(T)\right\|_{V_{1}}^{2}-\left\|y_{n}(0)\right\|_{V_{1}}^{2}\right) .
\end{aligned}
$$

The sequence $\left\|y_{n}(0)\right\|_{V_{1}}$ is bounded by $\|y(0)\|_{V_{1}}$ since by Proposition 2.8 it holds that $y_{n}(0)=\left(\mathcal{P}_{V_{1, n}} y\right)(0)$. Because of $\left\|\mathcal{L}_{1} \mathcal{L}_{3} y_{n}\right\|_{\mathcal{V}_{1}^{*}}=\left\|\mathcal{L}_{3} y_{n}\right\|_{\mathcal{V}_{1}}=\left\|y_{n}\right\|_{\mathcal{V}_{3}}$, the proof is finished.

Proof of Remark 2.11. First, notice that the eigenvectors $\left(e_{n}\right)$ of $L_{1}^{-1} I_{1}$ are elements of $V_{4}$. Then proceeding as above we obtain

$$
\|f\|_{\mathcal{H}}^{2} \geq\left\|y_{n}^{\prime}+\mathcal{L}_{2} \mathcal{L}_{4} y_{n}\right\|_{\mathcal{H}}^{2}=\left\|y_{n}^{\prime}\right\|_{\mathcal{H}}^{2}+2\left(y_{n}^{\prime} \mid \mathcal{L}_{2} \mathcal{L}_{4} y_{n}\right)_{\mathcal{H}}+\left\|\mathcal{L}_{2} \mathcal{L}_{4} y_{n}\right\|_{\mathcal{H}}^{2} .
$$


Again, using analogous commutation rules as given in Proposition 2.8, we conclude that

$$
\begin{aligned}
\left(y_{n}^{\prime} \mid \mathcal{L}_{2} \mathcal{L}_{4} y_{n}\right)_{\mathcal{H}} & =\left(\mathcal{L}_{2} y_{n}^{\prime} \mid \mathcal{L}_{2} y_{n}\right)_{\mathcal{H}} \\
& =\frac{1}{2}\left(\left\|y_{n}(T)\right\|_{V_{2}}^{2}-\left\|y_{n}(0)\right\|_{V_{2}}^{2}\right) .
\end{aligned}
$$

Since $\left\|y_{n}(0)\right\|_{V_{2}}$ is bounded by $\|y(0)\|_{V_{2}}$, the assertion follows.

In order to prove Proposition 2.12 we make use of a general result by Kampowsky [45] on the continuous Fréchet differentiability in Lebesgue spaces and reduce the problem to this case.

TheOREM A.2. Let $\left(U_{i}\right)_{1 \leq i \leq n}, V$ be normed vector spaces, let $\Omega \subset \mathbb{R}^{N}$ be a measurable subset, and let $q,\left(p_{i}\right)_{1 \leq i \leq n}$ be numbers with $1 \leq q<p_{i} \leq \infty$ for all $1 \leq i \leq n$. We define $r_{i}:=\left(\frac{1}{q}-\frac{1}{p_{i}}\right)^{-1}$ (with $\left.\frac{1}{\infty}:=0\right)$ and the spaces $U:=U_{1} \times \cdots \times U_{n}, \mathcal{U}_{i}:=$ $L^{p_{i}}\left(\Omega ; U_{i}\right), \mathcal{U}:=\mathcal{U}_{1} \times \cdots \times \mathcal{U}_{n}, \mathcal{V}:=L^{q}(\Omega ; V)$, and $\mathcal{W}_{i}:=L^{r_{i}}\left(\Omega ; \mathcal{L}\left(U_{i} ; V\right)\right)$ for all $1 \leq i \leq n$. Assume that the mapping $\gamma: \Omega \times U \rightarrow V$ possesses all partial derivatives $\partial_{u_{i}} \gamma=: \gamma_{i}: \Omega \times U \rightarrow \mathcal{L}\left(U_{i} ; V\right)$ in the $U_{i}$-variables as Fréchet derivatives, that these fulfill the Carathéodory condition, and that their superposition operators

$$
\left(A_{i} y\right)(x):=\gamma_{i}(x, y(x))
$$

map $\mathcal{U}$ continuously into $\mathcal{W}_{i}$. Then the superposition operator $A: \mathcal{U} \rightarrow \mathcal{V}$ of a is continuously Fréchet differentiable, and its derivative $A^{\prime}: \mathcal{U} \times \mathcal{L}(\mathcal{U} ; \mathcal{V})$ is given by

$$
\left(A^{\prime}(y ; v)\right)(x)=\sum_{i=1}^{n} A_{i}^{\prime}(y)(x)[v(x)]
$$

for all $y, v \in \mathcal{U}$ and almost all $x \in \Omega$.

Proof of Proposition 2.12. 1. In the first step we show that the superposition operator $A$ of $\gamma$

$$
(A y)(x):=\gamma(x, y(x))
$$

mapping $W^{1, s_{2}}(\Omega)$ into $H^{1}(\Omega)$ is continuously Fréchet differentiable, and its derivative $A^{\prime}: W^{1, s_{2}}(\Omega) \rightarrow \mathcal{L}\left(W^{1, s_{2}}(\Omega) ; H^{1}(\Omega)\right)$ is given by $A^{\prime}(y ; r)(x)=\gamma^{\prime}(y(x)) r(x)$.

For this purpose, choose $2<p_{1}<\infty$ such that $\frac{1}{2}-\frac{1}{s_{2}} \geq \frac{1}{p_{1}} \geq \frac{1}{s_{2}}-\frac{1}{N}$. The assumption guarantees that this is possible. Then, $W^{1, s_{2}}(\Omega)$ embeds continuously into $L^{p_{1}}(\Omega)$. Moreover,

$$
G: H^{1}(\Omega) \rightarrow L^{2}(\Omega) \times L^{2}\left(\Omega ; \mathbb{R}^{N}\right), \quad r \mapsto G r:=(y, \nabla y)
$$

is an isometric isomorphism from $H^{1}(\Omega)$ onto a closed subspace $H_{1}$ of the space $L^{2}(\Omega) \times L^{2}\left(\Omega ; \mathbb{R}^{N}\right)$, and the gradient $\nabla(\gamma \circ y)$ is given by $\gamma^{\prime}(y) \nabla y$. Instead of analyzing $A$ directly, we define the operator

$$
\begin{aligned}
\widetilde{A}: L^{p_{1}}(\Omega) \times L^{p_{2}}\left(\Omega ; \mathbb{R}^{N}\right) & \rightarrow L^{2}(\Omega) \times L^{2}\left(\Omega ; \mathbb{R}^{N}\right), \\
(y, v) & \mapsto\left(\gamma(y), \gamma^{\prime}(y) v\right)
\end{aligned}
$$

with $p_{2}:=s_{2}$ and use the fact that $A$ can be decomposed into

$$
\begin{aligned}
W^{1, s_{2}}(\Omega) & \rightarrow L^{p_{1}}(\Omega) \times L^{p_{2}}\left(\Omega ; \mathbb{R}^{N}\right) & \rightarrow \quad H_{1} & \rightarrow H^{1}(\Omega), \\
y & \mapsto \quad(y, \nabla y) & \mapsto \widetilde{A}(y, \nabla y) & \mapsto A y=G^{-1} \widetilde{A}(y, \nabla y) .
\end{aligned}
$$


Since $W^{1, s_{2}}(\Omega) \ni y \mapsto(y, \nabla y) \in L^{p_{1}}(\Omega) \times L^{p_{2}}\left(\Omega ; \mathbb{R}^{N}\right)$ and $G^{-1}: H_{1} \rightarrow H^{1}(\Omega)$ are linear, continuous operators, it suffices to prove that $\widetilde{A}$ is continuously Fréchet differentiable, and $\widetilde{A^{\prime}}: L^{p_{1}}(\Omega) \times L^{p_{2}}\left(\Omega ; \mathbb{R}^{N}\right) \rightarrow \mathcal{L}\left(L^{p_{1}}(\Omega) \times L^{p_{2}}\left(\Omega ; \mathbb{R}^{N}\right) ; L^{2}(\Omega) \times L^{2}\left(\Omega ; \mathbb{R}^{N}\right)\right)$ is given by

$$
\left(\widetilde{A}^{\prime}(y, v)[r, s]\right)(x)=\left(\gamma^{\prime}(y) r, \gamma^{\prime \prime}(y) r v+\gamma^{\prime}(y) s\right),
$$

because then we have

$$
\widetilde{A}^{\prime}(y, \nabla y)[r, \nabla r]=\left(\gamma^{\prime}(y) r, \nabla\left(\gamma^{\prime}(y) r\right)\right)=G\left(\gamma^{\prime}(y) r\right) .
$$

From the fact that $\gamma$ is differentiable and Lipschitz continuous, it follows that $y \mapsto$ $a(y)$ is continuously Fréchet differentiable from $L^{p_{1}}(\Omega)$ into $L^{2}(\Omega)$ by Theorem A.2 with derivative $\gamma^{\prime}(y)$. Moreover, the mapping $b:(f, h) \mapsto \gamma^{\prime}(f) h$ from $\mathbb{R} \times \mathbb{R}^{N}$ into $\mathbb{R}^{N}$ possesses the total differential $(D b)(e, g) \in \mathcal{L}\left(\mathbb{R} \times \mathbb{R}^{N} ; \mathbb{R}^{N}\right),(D b)(e, g)[f, h]=$ $\gamma^{\prime \prime}(e) f g+\gamma^{\prime}(e) h$. We know that $\gamma^{\prime \prime}(\mathbb{R})$ and $\gamma^{\prime}(\mathbb{R})$ are bounded subsets of $\mathbb{R}$, that $D b$ is continuous and

$$
\begin{aligned}
\left\|\left(\partial_{u} b\right)(e, g)\right\|_{\mathcal{L}\left(\mathbb{R} ; \mathbb{R}^{N}\right)} & =\left|\gamma^{\prime \prime}(e) g\right| \leq C|g| \\
\left\|\left(\partial_{v} b\right)(e, g)\right\|_{\mathcal{L}\left(\mathbb{R}^{N} ; \mathbb{R}^{N}\right)} & =\left|\gamma^{\prime}(e)\right| \leq C .
\end{aligned}
$$

Consequently, the corresponding superposition operators $B_{u}, B_{v}$ satisfy

$$
\begin{aligned}
& B_{u}: L^{p_{1}}(\Omega) \times L^{p_{2}}\left(\Omega ; \mathbb{R}^{N}\right) \rightarrow L^{p_{2}}\left(\Omega ; \mathcal{L}\left(\mathbb{R} ; \mathbb{R}^{N}\right)\right) \hookrightarrow L^{r_{1}}\left(\Omega ; \mathcal{L}\left(\mathbb{R} ; \mathbb{R}^{N}\right)\right), \\
& B_{v}: L^{p_{1}}(\Omega) \times L^{p_{2}}\left(\Omega ; \mathbb{R}^{N}\right) \rightarrow L^{\infty}\left(\Omega ; \mathcal{L}\left(\mathbb{R} ; \mathbb{R}^{N}\right)\right) \hookrightarrow L^{r_{2}}\left(\Omega ; \mathcal{L}\left(\mathbb{R}^{N} ; \mathbb{R}^{N}\right)\right)
\end{aligned}
$$

and are continuous with $p_{2}=s_{2} \geq r_{1}:=\left(\frac{1}{2}-\frac{1}{p_{1}}\right)^{-1}$ and $\infty \geq r_{2}:=\left(\frac{1}{2}-\frac{1}{p_{2}}\right)^{-1}$. Theorem A.2 now implies the continuous Fréchet differentiability of $A$ from $W^{1, s_{2}}(\Omega)$ into $H^{1}(\Omega)$ with the derivative as desired.

2. Now let us consider the superposition operator $\mathcal{A}$ of $A$ itself and show its continuous Fréchet differentiability. The superposition operator of $A^{\prime}$ will be denoted by $B$, i.e., $(B u)(t)=A^{\prime}(u(t))$. If we are able to show that $B$ maps $L^{s_{1}}\left(\mathcal{T} ; W^{1, s_{2}}(\Omega)\right)$ continuously into $L^{r}\left(\mathcal{T} ; \mathcal{L}\left(W^{1, s_{2}}(\Omega) ; H^{1}(\Omega)\right)\right)$ with $\frac{1}{s_{1}}+\frac{1}{r}=\frac{1}{2}$, then Theorem A.2 applies and we are done.

Consider $M:=\left\{y \in W^{1, s_{2}}(\Omega):\|y\|_{W^{1, s_{2}}(\Omega)} \leq 1\right\}$, and for $y, r \in W^{1, s_{2}}(\Omega)$

$$
\begin{aligned}
\left\|A^{\prime}(y ; r)\right\|_{H^{1}(\Omega)}^{2} & =\left\|\gamma^{\prime}(y) r\right\|_{H^{1}(\Omega)}^{2} \\
& =\left\|\gamma^{\prime}(y) r\right\|_{L^{2}(\Omega)}^{2}+\left\|\gamma^{\prime \prime}(y) r \nabla y\right\|_{L^{2}(\Omega)}^{2}+\left\|\gamma^{\prime}(y) \nabla r\right\|_{L^{2}(\Omega)}^{2} \\
& \leq C\left(\|r\|_{L^{2}(\Omega)}^{2}+\|r \nabla y\|_{L^{2}(\Omega)}^{2}+\|\nabla r\|_{L^{2}(\Omega)}^{2}\right) .
\end{aligned}
$$

The second summand can be estimated with the help of the generalized Hölder's inequality $\left(\|f g\|_{L^{p}} \leq\|f\|_{L^{q}}\|g\|_{L^{r}}\right.$ for $\left.\frac{1}{p}=\frac{1}{q}+\frac{1}{r}\right)$ and the Sobolev embedding $W^{1, s_{2}}(\Omega) \hookrightarrow L^{p_{1}}(\Omega)$ by

$$
\|r \nabla y\|_{L^{2}(\Omega)} \leq\|r\|_{L^{p_{1}(\Omega)}}\|\nabla y\|_{L^{q_{1}}(\Omega)} \leq C\|r\|_{W^{1, s_{2}(\Omega)}}\|y\|_{W^{1, s_{2}(\Omega)}}
$$

with $q_{1}:=\left(\frac{1}{2}-\frac{1}{p_{1}}\right)^{-1} \leq\left(\frac{1}{2}-\left(\frac{1}{2}-\frac{1}{s_{2}}\right)\right)^{-1}=s_{2}$ by the choice of $p_{1}$. Consequently, it holds that

$$
\left\|A^{\prime}(y)\right\|_{\mathcal{L}\left(W^{1, s_{2}}(\Omega) ; H^{1}(\Omega)\right)}=\sup \left\{\left\|A^{\prime}(y ; r)\right\|_{H^{1}(\Omega)}: r \in M\right\} \leq C\left(1+\|y\|_{W^{1, s_{2}}(\Omega)}\right) .
$$


Finally, we have $r \leq s_{1}$ by assumption, which implies that the operator

$$
B: L^{s_{1}}\left(\mathcal{T} ; W^{1, s_{2}}(\Omega)\right) \rightarrow L^{r}\left(\mathcal{T} ; \mathcal{L}\left(W^{1, s_{2}}(\Omega) ; H^{1}(\Omega)\right)\right)
$$

is continuous. This finishes the proof.

\section{REFERENCES}

[1] H. ABELS, Existence of weak solutions for a diffuse interface model for viscous, incompressible fluids with general densities, Comm. Math. Phys., 289 (2009), pp. 45-73.

[2] H. Abels and M. Wilke, Convergence to equilibrium for the Cahn-Hilliard equation with a logarithmic free energy, Nonlinear Anal. Theory Methods Appl., 67 (2007), pp. 3176-3193.

[3] Y. ACHDOU, An inverse problem for a parabolic variational inequality arising in volatility calibration with American options, SIAM J. Control Optim., 43 (2005), pp. 1583-1615.

[4] Y. Achdou and O. Pironneau, Computational Methods for Option Pricing, Frontiers Appl. Math. 30, SIAM, Philadelphia, 2005.

[5] R.A. Adams and J.J.F. Fournier, Sobolev Spaces, 2nd ed., Pure Appl. Math. 140, Academic Press, New York, 2003.

[6] H. Amann, Compact embeddings of vector-valued Sobolev and Besov spaces, Glas. Mat. Ser. III, 35 (2000), pp. 161-177.

[7] D.M. Anderson, G.B. McFadden, and A.A. Wheeler, Diffuse-interface methods in fluid mechanics, An. Rev. Fluid Mech., 30 (1998), pp. 139-165.

[8] L. Baňas and R. Nürnberg, Adaptive finite element methods for Cahn-Hilliard equations, J. Comput. Appl. Math., 218 (2008), pp. 2-11.

[9] L. BAŇAS AND R. NÜRnBERG, A multigrid method for the Cahn-Hilliard equation with obstacle potential, Appl. Math. Comput., 213 (2009), pp. 290-303.

[10] V. BArbu, Nonlinear Semigroups and Differential Equations in Banach Spaces, Editura Academiei Republicii Socialiste România, Bucharest, 1976.

[11] V. Barbu, Optimal Control of Variational Inequalities, Res. Notes Math. 100, Pitman (Advanced Publishing Program), Boston, 1984.

[12] V. Barbu and Th. Precupanu, Convexity and Optimization in Banach Spaces, 2nd rev. and extended ed., D. Reidel Publishing Company, Dordrecht/Boston/Lancaster, 1986.

[13] M. Bergounioux, Optimal control of problems governed by abstract elliptic variational inequalities with state constraints, SIAM J. Control Optim., 36 (1998), pp. 273-289.

[14] M. Bergounioux, Optimal control of semilinear elliptic obstacle problems, J. Nonlinear Convex Anal., 3 (2002), pp. 25-39.

[15] A. Bertozzi, S. Esedō̄uu, And A. Gillette, Analysis of a two-scale Cahn-Hilliard model for binary image inpainting, Multiscale Model. Simul., 6 (2007), pp. 913-936.

[16] L. Blank, M. Butz, And H. Garcke, Solving the Cahn-Hilliard Variational Inequality with a Semi-Smooth Newton Method, ESAIM Control Optim. Calc. Var., 17 (2011), pp. 931-954.

[17] J.F. Blowey AND C.M. Elliott, The Cahn-Hilliard gradient theory for phase separation with non-smooth free energy. I: Mathematical analysis, Eur. J. Appl. Math., 2 (1991), pp. 233-280.

[18] J.F. Blowey AND C.M. Elliott, The Cahn-Hilliard gradient theory for phase separation with non-smooth free energy. II: Numerical analysis, Eur. J. Appl. Math., 3 (1992), pp. 147-179.

[19] E. Bonetti, P. Colli, W. Dreyer, G. Gilardi, G. Schimperna, and J. Sprekels, On a model for phase separation in binary alloys driven by mechanical effects, Phys. D, 165 (2002), pp. 48-65.

[20] H. BRÉzIS, Opérateurs Maximaux Monotones et Semi-groupes de Contractions dans les Espaces de Hilbert, North-Holland Mathematics Studies 5, North-Holland, Amsterdam, 1973.

[21] J.W. Cahn And J.E. Hilliard, Free energy of a nonuniform system. I. Interfacial free energy, J Chem. Phys., 28 (1958), pp. 258-266.

[22] I. Capuzzo Dolcetta, S. Finzi Vita, and R. March, Area preserving curve-shortening flows: From phase separation to image processing, Interfaces and Free Bound., 4 (2002), pp. 325343.

[23] F.H. Clarke, Optimization and Nonsmooth Analysis, 2nd ed., Classics Appl. Math. 5, SIAM, Philadelphia, 1990.

[24] P. Colli, P. Krejčí, E. Rocca, And J. Sprekels, Nonlinear evolution inclusions arising from phase change models, Czech. Math. J., 57 (2007), pp. 1067-1098.

[25] M. Copetti And C.M. Elliott, Kinetics of phase decomposition processes: Numerical solutions to the Cahn-Hilliard equation, Materials Science and Technology, 6 (1990), pp. $273-283$. 
[26] J. Diestel And J.J. Jun. Uhl, Vector Measures, Math. Surveys 15, AMS, Providence, RI, 1977.

[27] C.M. Elliott and S. Zheng, On the Cahn-Hilliard equation, Arch. Ration. Mech. Anal., 96 (1986), pp. 339-357.

[28] H.B. Frieboes et AL., Towards multiscale modeling of nanovectored delivery of therapeutics to cancerous lesions, Biomed. Dev., to appear.

[29] H. Gajewski, K. Gröger, and K. Zacharias, Nichtlineare Operatorgleichungen und Operatordifferentialgleichungen, Mathematische Lehrbücher und Monographien 38, AkademieVerlag, Berlin, 1974.

[30] H. Garcke, On Cahn-Hilliard systems with elasticity, Proc. R. Soc. Edinburgh Sect. A Math., 133 (2003), pp. 307-331.

[31] H. Garcke, Optimization Problems and Cahn-Hilliard Systems, Technical report, Mathematisches Institut Oberwolfach, Oberwolfach-Walke, Germany, 2007.

[32] R. Glowinski and A.M. Ramos, A numerical approach to the Neumann control of the CahnHilliard equation, in Computational Methods for Control and Applications, Gakuto International Series: Mathematical Sciences and Applications 16, Gakkotosho Co., Tokyo, 2002, pp. 111-155.

[33] C. Gräser And R. Kornhuber, On preconditioned Uzawa-type iterations for a saddle point problem with inequality constraints, in Domain Decomposition Methods in Science and Engineering XVI, Lect. Notes Comput. Sci. Eng. 55, Springer, Berlin, 2007, pp. 91-102.

[34] C. Gräser and R. Kornhuber, Multigrid methods for obstacle problems, J. Comput. Math., 27 (2009), pp. 1-44.

[35] P. Grisvard, Elliptic Problems in Nonsmooth Domains, Monogr. Stud. Math. 24, Pitman (Advanced Publishing Program), Boston, 1985.

[36] K. GröGER, Lecture on 'Evolutionsgleichungen,' Humboldt-Universität Berlin, Berlin, 2001.

[37] F. Hausser, S. Rasche, And A. Voigt, The influence of electric fields on nanostructuressimulation and control, Math. Comput. Simulation, 80 (2010), pp. 1449-1457.

[38] M. HintermüLler, Inverse coefficient problems for variational inequalities: Optimality conditions and numerical realization, M2AN Math. Model. Numer. Anal., 35 (2001), pp. 129152.

[39] M. HintermüLler, An active-set equality constrained Newton solver with feasibility restoration for inverse coefficient problems in elliptic variational inequalities, Inverse Problems, 24 (2008), 034017.

[40] M. Hintermüller, M. Hinze, and M.H. Tber, An adaptive finite element Moreau-Yosidabased solver for a non-smooth Cahn-Hilliard problem, Optim. Methods Softw., 26 (2011), pp. $777-811$.

[41] M. HintermüLler AND I. KopaCKA, Mathematical programs with complementarity constraints in function space: $C$ - and strong stationarity and a path-following algorithm, SIAM J. Optim., 20 (2009), pp. 868-902.

[42] M. Hintermüller AND I. KopaCKA, A smooth penalty approach and a nonlinear multigrid algorithm for elliptic MPECs, Comput. Optim. Appl., 50 (2011), pp. 111-145.

[43] M. Hintermüller And M.H. Tber, An inverse problem in American options as a mathematical program with equilibrium constraints: $C$-stationarity and an active-set-Newton solver, SIAM J. Control Optim., 48 (2010), pp. 4419-4452.

[44] K. ITǑ And K. KunisCh, Optimal control of obstacle problems by $H^{1}$-obstacles, Appl. Math. Optim., 56 (2007), pp. 1-17.

[45] W. Kampowsky, Notwendige Optimalitätsbedingungen für Prozesse in Evolutionsgleichungen, Inaugural-Dissertation, Universität Greifswald, Greifswald, Germany, 1979.

[46] N. Kenmochi, M. NiezgódKa, And I. PawŁow, Subdifferential operator approach to the CahnHilliard equation with constraint, J. Differential Equations, 117 (1995), pp. 320-356.

[47] Z.-Q. LuO, J.-S. PAng, And D. RAlPh, Mathematical Programs with Equilibrium Constraints, Cambridge University Press, Cambridge, UK, 1997.

[48] M. Marcus And V.J. Mizel, Every superposition operator mapping one Sobolev space into another is continuous, J. Funct. Anal., 33 (1979), pp. 217-229.

[49] H. MaUrer And J. Zowe, First and second order necessary and sufficient optimality conditions for infinite-dimensional programming problems, Math. Programming, 16 (1979), pp. 98110.

[50] B.S. Mordukhovich, Variational Analysis and Generalized Differentiation, I Basic Theory, Grundlehren Math. Wiss. 330, Springer-Verlag, Berlin, 2006.

[51] B.S. Mordukhovich, Variational Analysis and Generalized Differentiation. II Applications, Grundlehren Math. Wiss. 331, Springer-Verlag, Berlin, 2006. 
[52] P. Neittanmaki, J. Sprekels, and D. Tiba, Optimization of Elliptic Systems. Theory and Applications, Springer Monogr. Math., Springer, New York, 2006.

[53] P. Neittananmïr and D. Tiba, Optimal Control of Nonlinear Parabolic Systems: Theory, Algorithms, and Applications, Pure Appl. Math., Marcel Dekker, New York, 1994.

[54] A. Novick-Cohen And L.A. Segel, A gradually slowing travelling band of chemotactic bacteria, J. Math. Biol., 19 (1984), pp. 125-132.

[55] Y. Oono And S. Puri, Study of phase-separation dynamics by use of cell dynamical systems. I. Modeling, Phys. Rev. A, 38 (1988), pp. 434-453.

[56] J. Outrata, M. KoČvara, And J. Zowe, Nonsmooth Approach to Optimization Problems with Equilibrium Constraints. Theory, Applications and Numerical Results, Nonconvex Optim. Appl. 28, Kluwer Academic Publishers, Dordrecht, 1998.

[57] H. Scheel And S. Scholtes, Mathematical programs with complementarity constraints: Stationarity, optimality, and sensitivity, Math. Oper. Res., 25 (2000), pp. 1-22.

[58] I. Steinbach, Phase-field models in materials science, Modelling and Simulation in Materials Science and Engineering, 17 (2009), 073001.

[59] H. Triebel, Interpolation Theory, Function Spaces, Differential Operators, 2nd revised and enlarged ed., Johann Ambrosius Barth, Heidelberg, 1995.

[60] Q.-F. WANG AND S. NAKAGIRI, Weak solutions of Cahn-Hilliard equations having forcing terms and optimal control problems, in Mathematical Models in Functional Equations (Kyoto, 1999), Research Institute for Mathematical Sciences 1128, Kyoto University, Kyoto, 2000, pp. 172-180 (in Japanese).

[61] D. Wegner, Solvability of Pseudomonotone-Strongly Monotone Systems and Application to a Model of Phase Separation, Ph.D. thesis, Humboldt Universität zu Berlin, Berlin, 2010.

[62] J. Yong And S. Zheng, Feedback stabilization and optimal control for the Cahn-Hilliard equation, Nonlinear Anal., 17 (1991), pp. 431-444.

[63] J. Zowe And S. Kurcyusz, Regularity and stability for the mathematical programming problem in Banach spaces, Appl. Math. Optim., 5 (1979), pp. 49-62. 